\title{
Hierarchical Modeling and Analysis of Embedded Systems
}

\author{
RAJEEV ALUR, MEMBER, IEEE, THAO DANG, JOEL ESPOSITO, YERANG HUR, \\ FRANJO IVANČIĆ, STUDENT MEMBER, IEEE, VIJAY KUMAR, INSUP LEE, FELLOW, IEEE, \\ PRADYUMNA MISHRA, GEORGE J. PAPPAS, MEMBER, IEEE, AND OLEG SOKOLSKY
}

\section{Invited Paper}

This paper describes the modeling language CHARON for modular design of interacting hybrid systems. The language allows specification of architectural as well as behavioral hierarchy and discrete as well as continuous activities. The modular structure of the language is not merely syntactic, but is exploited by analysis tools and is supported by a formal semantics with an accompanying compositional theory of refinement. We illustrate the benefits of CHARON in the design of embedded control software using examples from automated highways concerning vehicle coordination.

Keywords-Embedded control systems, formal analysis tools, hybrid systems, modular design.

\section{INTRODUCTION}

An embedded system typically consists of a collection of digital programs that interact with each other and with an analog environment. Examples of embedded systems include manufacturing controllers, automotive controllers, engine controllers, avionic systems, medical devices, microelectromechanical systems, and robots. As computing tasks performed by embedded devices become more sophisticated, the need for a sound discipline for writing embedded software becomes more apparent (cf. [1]). An embedded system consisting of sensors, actuators, plant, and control software is best viewed as a hybrid system. The relevance of hybrid modeling has been demonstrated in various applications such as coordinating robot systems [2], automobiles [3], aircraft [4],

Manuscript received December 20, 2001; revised August 31, 2002. This work was supported in part by the Defense Advanced Research Projects Agency under Model-Based Integrated Embedded Software Contract F33615-00-C-1707; in part by the Army Research Office under Contract DAAD19-01-1-0473; in part by the National Science Foundation under Grant CCR-9970925, Grant CCR-9988409, Grant CCR-0086147, Grant CISE-9703220, and Grant ITR CCR01-21431; and in part by the Office of Naval Research under Contract N00014-97-1-0505.

The authors are with the Department of Computer and Information Science, University of Pennsylvania, Philadelphia, PA 19104, USA.

Digital Object Identifier 10.1109/JPROC.2002.805817 and chemical process control systems [5]. A model-based design paradigm is particularly attractive because of its promise for greater design automation and formal guarantees of reliability.

Traditionally, control theory and related engineering disciplines have addressed the problem of designing robust control laws to ensure optimal performance of processes with continuous dynamics. This approach to system design largely ignores the problem of implementing control laws as a piece of software and issues related to concurrency and communication. Computer science and software engineering, on the other hand, have an entirely discrete view of the world, which abstracts from the physical characteristics of the environment to which the software is reacting and is typically unable to guarantee safety and/or performance of the embedded device as a whole. Hybrid modeling combines these two approaches and is natural for specification of embedded systems.

We have been developing a modeling language, CHARON, that is suitable for specification of interacting embedded systems as communicating agents. CHARON has been used in the modeling and analysis of a wide range of hybrid systems, such as automotive power trains, vehicle-to-vehicle control systems [6], biological cells [7], multiagent systems [8], [9], and infusion pump and inverted pendulum systems [10]. The two salient aspects of CHARON are that it supports modular specifications and that it has a well-defined formal semantics.

Hierarchical, Modular Modeling: Modern software design paradigms promote hierarchy as one of the key constructs for structuring complex specifications. They are concerned with two distinct notions of hierarchy. In architectural hierarchy, a system with a collection of communicating agents is constructed by parallel composition of atomic agents; in behavioral hierarchy, the behavior of an individual agent is described by hierarchical sequential composition. The former 
hierarchy is present in almost all concurrency formalisms, and the latter, while present in all block-structured programming languages, was introduced for state-machine-based modeling in STATECHARTS [11]. CHARON supports both architectural and behavioral hierarchies.

Early formal models for hybrid systems include phase transition systems [12] and hybrid automata [13]. Although modularity in hybrid specifications has been addressed in languages such as hybrid input-output (I/O) automata [14], CHARON allows richer specifications. Discrete updates in CHARON are specified by guarded actions labeling transitions connecting the modes. Some of the variables in CHARON can be declared analog, and they flow continuously during continuous updates that model the passage of time. The evolution of analog variables can be constrained in three ways: differential constraints (e.g., by equations such as $\dot{x}=f(x, u)$ ), algebraic constraints (e.g., by equations such as $y=g(x, u)$ ), and invariants (e.g., $|x-y| \leq \varepsilon$ ) which limit the allowed durations of flows.

Compositional Semantics: Formal semantics leads to definitions of semantic equivalence (or refinement) of specifications based on their observable behaviors. Compositional here means that semantics of a component can be constructed from the semantics of its subcomponents. Such formal compositional semantics is a cornerstone of concurrency frameworks such as communicating sequential processes (CSP) [15] and the calculus of communicating systems (also referred to as CCS) [16], and is a prerequisite for developing modular reasoning principles such as compositional model checking and systematic design principles such as stepwise refinement.

Two aspects of CHARON make it difficult to adopt existing techniques. First, the global nature of time makes it challenging to define semantics of hybrid components in a modular fashion. Second, features such as group transitions, exceptions, and history retention supporting rich hierarchical specifications cause additional difficulties. The compositional semantics of CHARON supports observational trace semantics for both modes and agents [17]. The key result is that the set of traces of a mode can be constructed from the traces of its submodes. This result leads to a compositional notion of refinement for modes.

This paper is organized as follows. Section II gives a short overview of related work. In Section III, we present the features of the language CHARON, and in Section IV we describe the formal semantics and accompanying compositional refinement calculus, using examples from the automotive experimental platform of DARPA's MoBIES project. Section $\mathrm{V}$ gives an overview of ongoing research on formal analysis. We conclude in Section VI with a summary of the CHARON design toolkit.

\section{BACKGROUND}

Software Design Notations: Modern object-oriented design paradigms such as the Unified Modeling Language (UML) allow specification of the architecture and control at high levels of abstraction in a modular fashion and bear great promise as a solution to managing the complexity at all stages of the software design cycle [18]. Emerging tools such as RationalRose (available: www.rational.com) support modeling, simulation, and code generation and are becoming increasingly popular in domains such as automotive software and avionics.

Tool Support for Control System Design: Traditionally, control engineers have used tools for continuous differential equations such as MATLAB (available: www.mathworks.com) for modeling of the plant behavior, for deriving and optimizing control laws, and for validating functionality and performance of the model through analysis and simulation. Tools such as SIMULINK recently augmented the continuous modeling with state-machine-based modeling of discrete control.

Modeling Languages for Hybrid Systems: To benefit from object-oriented design, several languages that support objectoriented modeling of complex dynamical systems have been proposed. Omola [19], Dymola [20], and Modelica [21] provide noncausal models; that is, there is no notion of causality in the equations in the models. Those three have been used mostly for describing physical objects, whereas SHIFT [22] is more like a programming language and has been used extensively to specify automated vehicle highway systems. PTOLEMY II [23] supports the modeling, simulation, and design of concurrent systems. It incorporates a number of models of computation (such as synchronous/reactive systems, CSP, finite state machines, continuous time, etc) with semantics that allow domains to interoperate.

All the above languages were proposed for modeling and simulation purposes and have not been used for formal verification of systems. CHARON has compositional formal semantics required to reason about systems in a modular way while incorporating many features of the aforementioned languages. Two features that are not supported by CHARON are model inheritance and dynamic creation of model instances.

Model Checking: Inspired by the success of model checking in hardware verification and protocol analysis [24], [25], there has been increasing research on developing techniques for automated verification of hybrid (mixed discrete-continuous) models of embedded controllers [13], [26]-[29]. The state-of-the-art computational tools for model checking of hybrid systems are of two kinds. Tools such as KRONOS [30], UPPAAL [31], and HYTECH [32] limit the continuous dynamics to simple abstractions such as rectangular inclusions (e.g., $\dot{x} \in[1,2]$ ) and compute the set of reachable states exactly and effectively by symbolic manipulation of linear inequalities. On the other hand, emerging tools such as CHECKMATE [33], d/dt [34], and level-sets method [35], [36] approximate the set of reachable states by polyhedra or ellipsoids [37] using optimization techniques. Even though these tools have been applied to interesting real-world examples after appropriate abstractions, scalability remains a challenge.

\section{Modeling LANGUAGE}

In CHARON, the building block for describing the system architecture is an agent that communicates with its environ- 
ment by means of shared variables. The language supports the operations of composition of agents to model concurrency, hiding of variables to restrict sharing of information, and instantiation of agents to support reuse. The building block for describing flow of control inside an atomic agent is a mode. A mode is basically a hierarchical state machine; that is, a mode can have submodes and transitions connecting them. Variables can be declared locally inside any mode with standard scoping rules for visibility. Modes can be connected to each other only by well-defined entry and exit points. We allow sharing of modes so that the same mode definition can be instantiated in multiple contexts. To support exceptions, the language allows group transitions from default exit points that are applicable to all enclosing modes; to support history retention, the language allows default entry transitions that restore the local state within a mode from the most recent exit.

Case Study: Throughout this paper, we will use a recent case study to illustrate the modeling and analysis concepts within the proposed framework. The case study is based on the longitudinal control system for vehicles moving in an Intelligent Vehicle Highway System (IVHS) [38]. A detailed description of the system can be found in [39]. Before proceeding with the modeling of the problem, we present a brief informal description of the control system.

In the context of IVHS, vehicles travel in platoons; inside a platoon, all vehicles follow the leader. We consider a platoon $i$ and its preceding platoon $(i-1)$. Let $v_{i}$ and $a_{i}$ denote the velocity and acceleration, respectively, of the platoon $i$, and let $d_{i}$ be its distance to the platoon $(i-1)$. The most important task of a longitudinal controller for the leader car of each platoon $i$ is to maintain the distance $d_{i}$ equal to a safety distance $D_{i}=\lambda_{a} a_{i}+\lambda_{v} v_{i}+\lambda_{p}$; in the nominal operation, $\lambda_{a}=0 s^{2}, \lambda_{v}=1 s$ and $\lambda_{p}=10 \mathrm{~m}$. Other tasks the controller should perform are to track an optimal velocity and trajectories for certain maneuvers. Without going into details, the controller for the leader car of platoon $i$ proposed in [39] consists of four control laws $u$, which are used in different regions of the state space. These regions are defined based on the values of the relative velocity $v_{i}^{e}=100\left(v_{i-1}-\right.$ $\left.v_{i}\right) / v_{i}$ and the error between the actual and the safe interplatoon distances $e_{i}=d_{i}-D_{i}$. When $v_{i}^{e}$ and $e_{i}$ change from one region to another, the control law should change accordingly. One important property we want to verify is that a collision between platoons never happens, that is, $d_{i}>$ $0 \mathrm{~m}$. To this end, we consider a system with four continuous variables $\left(d_{i}, v_{i-1}, v_{i}, a_{i}\right)$. The dynamics of these variables are as follows:

$$
\begin{cases}\dot{d}_{i} & =v_{i-1}-v_{i} \\ \dot{v}_{i-1} & =a_{i-1} \\ \dot{v}_{i} & =a_{i} \\ \dot{a}_{i} & =u\end{cases}
$$

where $u$ is the control. One can see that the dynamics of each platoon depends on the state of its preceding platoon. We consider a pair of platoons $(i-1)$ and $i$ and prove that the controller of the leader car of platoon $i$ can guarantee that no collision happens regardless of the behavior of platoon $(i-1)$. More precisely, the acceleration $a_{i-1}$ of the platoon in front is treated as uncertain input with values in the interval [ $\left.a_{\min }, a_{\max }\right]$ where $a_{\min }$ and $a_{\max }$ are the maximal possible deceleration and acceleration.

\section{A. Agents and Architectural Hierarchy}

The architectural hierarchy of the above platoon control system is shown in Fig. 1. The agent PLATOON- $i$ consists of two subagents, namely VELOCITY and CONTROLLER. The subagent CONTROLLER models the control laws and outputs the acceleration $a_{i}$ of the platoon $i$. The subagent VELOCITY takes as input the variable acc and updates the variable vel of the platoon $i$. The agent PLATOON- $(i-1)$, whose role is to model all possible behaviors of the platoon in front, outputs its own velocity (variable vel) to the agent PLATOON- $i$. In other words, the velocity (or acceleration) of the platoon $(i-1)$ can be seen as uncertain input (or external disturbance) to the agent PLATOON- $i$.

Each agent has a well-defined interface consisting of its typed input and output variables, represented visually as blank and filled squares, respectively. The two variables vel of the agents PLATOON- $(i-1)$ and PLATOON- $i$ are inputs to the agent DISTANCE, which outputs the distance between the two platoons. The subagent CONTROLLER of PLATOON- $i$ computes the desired acceleration $a_{i}$ based on the inter-platoon distance and the velocity of the platoon in front.

Formally, an agent, $A=\langle T M, V, I\rangle$, consists of a set $V$ of variables, a set $I$ of initial states and a set $T M$ of modes. The set $V$ is partitioned into local variables $V_{l}$ and global variables $V_{g}$; global variables are further partitioned into input and output variables. Type correct assignments of values to variables are called valuations and denoted $Q_{V}$. The set of initial states $I \subseteq Q_{V}$ specifies possible initializations of the variables of the agent. The modes, described in more detail below, collectively define the behavior of the agent. An atomic agent has a single top-level mode. Composite agents are constructed from other agents and have many top-level modes. For example, the behavior of the agent PLATOON- $i$ is given by the top-level modes of its atomic subagents, VELOCITY and CONTROLLER.

Fig. 1 illustrates the three operations defined on agents. Agents can be composed in parallel with each other. The parallel agents execute concurrently and communicate through shared variables. To enable communication between agents, global variables are renamed. For example, variables vel of agents PLATOON- $(i-1)$ and PLATOON- $i$ are renamed into velfirst and velother, respectively, so that the agent DISTANCE can read them without confusion. Finally, the communication between the vehicles can be hidden from the outside world. In our example, only the variable vel is the output of the PLATOON- $i$ agent. The variable acc, used internally by the agent PLATOON- $i$, cannot be accessed from the outside.

\section{B. Modes and Behavioral Hierarchy}

Modes represent behavioral hierarchy in the system design. The behavior of each atomic agent is described by a 


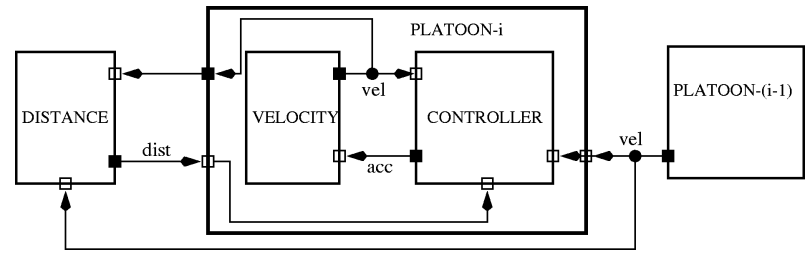

Fig. 1 The architectural hierarchy of the platoon controller.

mode, which corresponds to a single thread of discrete control. Each mode has a well-defined data interface consisting of typed global variables used for sharing state information and also a well-defined control interface consisting of entry and exit points, through which discrete control enters and exits the mode. Entry and exit points are denoted as blank and filled circles, respectively. A top-level mode, which is activated when the corresponding agent comes into existence and is never deactivated, has a special entry point init.

At the lowest level of the behavioral hierarchy are atomic modes. They describe continuous behaviors. For example, Fig. 2 illustrates the behavior of the mode Track, which specifies a control law by means of a differential constraint that asserts the relationship between desired acceleration acc and input variables of the mode, representing the velocities of the platoon, the platoon in front of it and the distance between platoons. CHARON also supports algebraic constraints on variable values. In addition, an invariant may be used to specify how long the mode can remain active. Once an invariant is violated, the mode has to be exited by taking one of the transitions leaving the mode.

The values of $\mathrm{k} 1, \mathrm{k} 2, \mathrm{k} 3$, lambdaP, and lambdaV are parameters of the mode. The mode can be instantiated with different values for the parameters several times in the same model, yielding different control laws. This will be illustrated later.

Composite modes contain a number of submodes. During execution, a composite mode performs discrete transitions that connect its control points and control points of its submodes. For example, the behavior of the agent Controller is captured by the mode shown in Fig. 3. To avoid cluttering the figure, we omit the guards on mode transitions.

Formally, a mode $M=\langle S M, V, E, X, T$, Cons $\rangle$ consists of a set of submodes $S M$, a set of variables $V$, a set of entry control points $E$, a set of exit control points $X$, a set of transitions $T$, and a set of constraints Cons. As in agents, variables are partitioned into global and local variables. For the submodes of $M$, we require that each global variable of a submode is a variable (either global or local) of $M$. This induces a natural scoping rule for variables in a hierarchy of modes: a variable introduced as local in a mode is accessible in all its submodes but not in any other mode. Every mode has two distinguished control points, called default entry $(d e)$ and exit $(d x)$ points. They are used to represent such high-level behavioral notions as interrupts and exceptions, which will be discussed in more detail in Section IV.

Constraints of a mode define continuous behavior of a mode in three ways. Continuous trajectories of a variable $x$

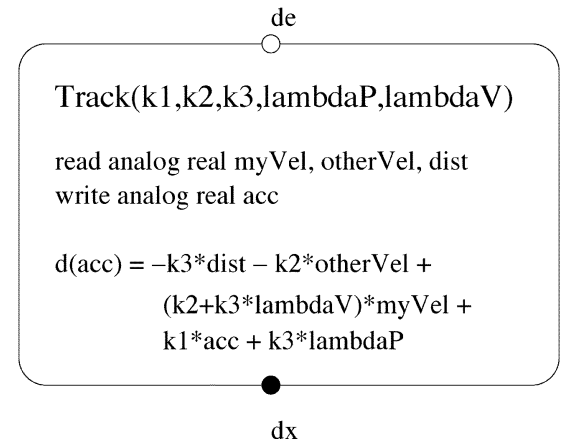

Fig. 2 Mode Track.

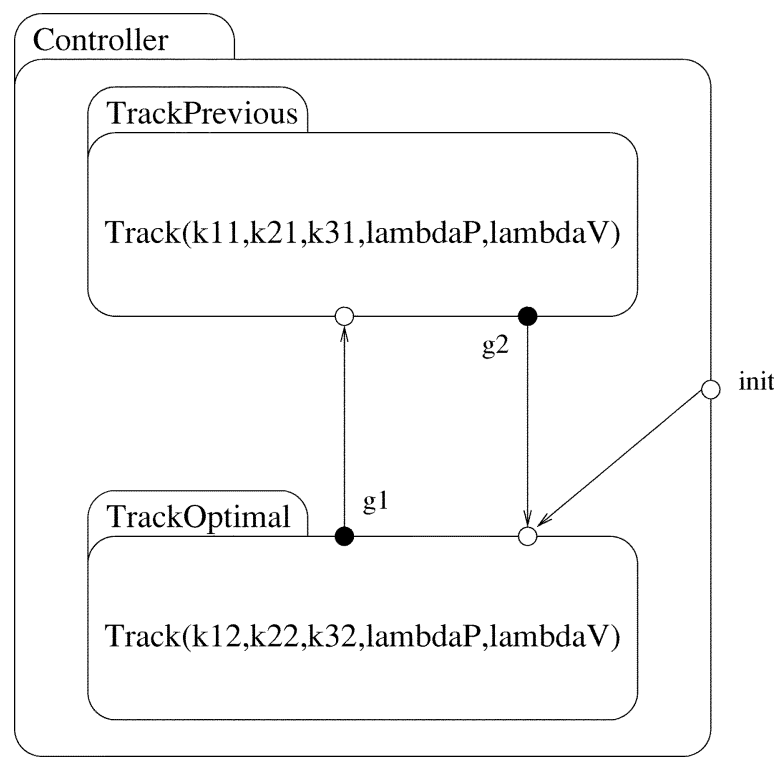

Fig. 3 Behavior of the agent Controller.

can be given by either an algebraic constraint $A_{x}$, which defines the set of admissible values for $x$ in terms of values of other variables, or by a differential constraint $D_{x}$, which defines the admissible values for the first derivative of $x$ with respect to time. Additionally, only those trajectories are allowed that satisfy the invariant of the mode, which is a predicate over the mode variables.

Transitions of a mode $M$ can be classified as entry transitions, which connect an entry point of $M$ with an entry point of one of its submodes; exit transitions, connecting exit points of submodes to exit points of $M$; and internal transitions that lead from an exit point of a submode to an entry point of another submode. In the example, the entry transition of Controller specifies that the mode starts in the TrackOptimal submode, which will be used to "catch up" with the platoon in front. There are no exit transitions, since it is a top-level mode and must execute forever. Every transition has a guard, which is a predicate over the valuations of mode variables that specifies when the transition can be executed. When a transition occurs, it executes a sequence of assignments, changing values of the mode variables. A transition that originates at a default exit point of a submode is called a group transition of that submode. A group transition can be executed to interrupt the execution of the submode. 
In CHARON, transitions and constraints can refer to externally defined Java classes, thus allowing richer discrete and continuous specifications.

\section{FORMAL SEMANTICS AND COMPOSITIONAL REFINEMENT}

In this section, we first define the operational semantics of modes and agents that makes the notion of executing a CHARON model precise and can be used, say, by a simulator. Second, we define observational semantics for modes and agents. The observational semantics hides the details about internal structure and retains only the information about inputs and outputs. Informally, the observational semantics consists of the static interface (such as the global variables and entry/exit points) and dynamic interface consisting of the traces, that is, sequences of updates to global variables. Third, for modularity, we show that our semantics is compositional. This means that the set of traces of a component can be defined from the set of traces of its subcomponents. Intuitively, this means that the observational semantics captures all the information that is needed to determine how a component interacts with its environment. Finally, we define a notion of refinement (or equivalence) for modes/agents. This allows us to relate different models of the same system. We can establish, for instance, that an abstract (simplified) version of a platoon refines a detailed version, and then analyze control of platoons using the abstract version instead of the detailed one, significantly simplifying analysis. The compositional rules about refinement form the basis for analysis in a system with multiple components, each with a simplified and a detailed model.

\section{A. Formal Semantics of Modes}

Intuitive Semantics: Before presenting the semantics formally, we give the intuition for mode executions. A mode can engage in discrete or continuous behavior. During an execution, the mode and its environment either take turns making discrete steps or take a continuous step together. Discrete and continuous steps of the mode alternate. During a continuous step, the mode follows a continuous trajectory that satisfies the constraints of the mode. In addition, the set of possible trajectories may be restricted by the environment of the mode. In particular, when the mode invariant is violated, the mode must terminate its continuous step and take one of its outgoing transitions. A discrete step of the mode is a finite sequence of discrete steps of the submodes and enabled transitions of the mode itself. A discrete step begins in the current state of the mode and ends when it reaches an exit point or when the mode decides to yield control to the environment and lets it make the choice of the next step. Technically, when the mode ends its discrete step in one of its submodes, it returns control to the environment via its default exit point. The closure construction, described later, ensures that the mode can yield control at appropriate moments and that the discrete control state of the mode is restored when the environment schedules the next discrete step.
Preemption: An execution of a mode can be preempted by a group transition. A group transition of a mode originates at the default exit of the mode. During any discrete step of the mode, control can be transferred to the default exit, and an enabled group transition can be selected. There is no priority between the transitions of a mode and its group transitions. When an execution of a mode is preempted, the control state of the mode is recorded in a special history variable, a new local variable that we introduce into every mode. Then, when the mode is entered through the default entry point next time, the control state of the mode is restored according to the history variable.

The History Variable and Active Submodes: To record the location of discrete control during executions, we introduce a new local variable $h$ into each mode that has submodes. The history variable $h$ of a mode $M$ has the names of the submodes of $M$ as values, or a special value $\epsilon$ that is used to denote that the mode is not active. A submode $N$ of $M$ is called active when the history variable of $M$ has the value $N$.

Flows: To precisely define continuous trajectories of a mode, we introduce the notion of a flow. A flow for a set $V$ of variables is a differentiable function $f$ from a closed interval of nonnegative reals $[0, \delta]$ to $Q_{V}$. We refer to $\delta$ as the duration of the flow. We denote a set of flows for $V$ as $\mathcal{F}_{V}$.

Syntactic Restrictions on Modes: To ensure that the semantics of a mode is well-defined, we impose several restrictions on mode structure. First, we assume that the set of differential and algebraic constraints in a mode always has a nonempty set of flows that satisfy them. This is needed to ensure that the set of behaviors of a mode is nonempty. Furthermore, we require that the mode cannot be blocked at any of its nondefault control points. This means that the disjunction of all guards originating from a control point evaluates to true.

State of a Mode: We define the state of a mode in terms of all variables of the mode and its submodes, including the local variables on all levels. We use $V_{*}$ for the set of all variables. The local variables of a mode together with the local variables of the submodes are called the private variables; this set of variables is denoted as $V_{p}$.

The state of a mode $M$ is a pair $(c, s)$, where $c$ is the location of discrete control in the mode and $s \in Q_{M . V_{*}}$. Whenever the mode has control, it resides in one of its control points, that is, $c \in M$.C. Given a state $(c, s)$ of $M$, we refer to $c$ as the control state of $M$ and to $s$ as the data state of $M$.

Closure of a Mode: Closure construction is a technical device to allow the mode to interrupt its execution and to maintain its history variable. Transitions of the mode are modified to update the history variable $h$ after a transition is executed. Each entry or internal transition assigns the name of the destination mode to $h$, and exit transitions assign $\epsilon$ to $h$. In addition, default entry and exit transitions are added to the set of transitions of the mode. These default transitions do not affect the history variable and allow us to interrupt an execution and then resume it later from the same point.

The default entry and exit transitions are added in the following way. For each submode $N$ of $M$, the closure adds a 
default exit transition from $N . d x$ to $M . d x$. This transition does not change any variables of the mode and is always enabled. Default entry transitions are used to restore the local control state of $M$. A default entry transition that leads from a default entry of $M$ to the default entry of a submode $N$ is enabled if $h=N$. Furthermore, we make sure that the default entry transitions do not interfere with regular entry transitions originating from $d e$. The closure changes each such transition so that it is enabled only if $h=\epsilon$. The closure construction for the mode Controller introduced in Section III-B is illustrated in Fig. 4.

Operational Semantics: An operational view of a closed-mode $M$ with the set of variables $V$ consists of a continuous relation $R^{C}$ and, for each pair $c_{1} \in E, c_{2} \in X$, a discrete relation $R_{c_{1}, c_{2}}^{D}$.

The relation $R^{C} \subseteq Q_{V} \times \mathcal{F}_{V}$ gives, for every data state of the mode, the set of flows from this state. By definition, if the control state of the mode is not at $d x$, the set of flows for the state is empty. $R^{C}$ is obtained from the constraints of a mode and relations $S M . R^{C}$ of its submodes. Given a data state $s$ of a mode $M,(s, f) \in R^{C}$ iff $f$ satisfies the constraints of $M$ and, if $N$ is the active submode at $s,(s, f)$, restricted to the global variables of $N$, belongs to N.R $R^{C}$.

The relation $R_{e, x}^{D}$, for each entry point $e$ and exit point $x$ of a mode, is composed of macrosteps of a mode starting at $e$ and ending at $x$. A macrostep consists of a sequence of microsteps. Each microstep is either a transition of the mode or a macrostep of one of its submodes. Given the relations $R_{e, x}^{D}$ of the submodes of $M$, a microexecution of a mode $M$ is a sequence of the form $\left(e_{0}, s_{0}\right),\left(e_{1}, s_{1}\right), \ldots,\left(e_{n}, s_{n}\right)$ such that every $\left(e_{i}, s_{i}\right)$ is a state of $M$ and for even $i$, $\left(\left(e_{i}, s_{i}\right),\left(e_{i+1}, s_{i+1}\right)\right)$ is a transition of $M$, while for odd $i,\left(s_{i}, s_{i+1}\right)$ is a macrostep of one of the submodes of $M$. Given such a microexecution of $M$ with $e_{0}=e \in E$ and $e_{n}=x \in X$, we have $\left(s_{0}, s_{n}\right) \in R_{e, x}^{D}$. To illustrate the notion of macrosteps, consider the closed-mode Controller from Fig. 4. Let $s$ be such that $h=\epsilon$ and $g_{1}$ is false. Then there is a microexecution for Controller : init, TrackOptimal.de, TrackOptimal.dx, and $d x$ (we show only the control points of the microexecution for clarity). This means that $(s, s[h:=$ TrackOptimal $]) \in$ $R_{\text {init, } d x}^{D}$. If $g_{1}$ is true in a state $s^{\prime}$, then $\left(s^{\prime}, s^{\prime}[h:=\right.$ TrackPrevious]) $\in R_{\text {init,dx }}^{D}$ corresponding to the microexecution init,Track0ptimal.de, TrackOptimal.dx, TrackOptimal.de, TrackOptimal. $d x$, and $d x$.

The operational semantics of the mode $M$ consists of its control points $E \cup X$, its variables $V$ and relations $R^{C}$ and $R_{e, x}^{D}$. The operational semantics of a mode defines a transition system $\mathcal{R}$ over the states of the mode. We write $\left(e_{1}, s_{1}\right) \stackrel{o}{\rightarrow}\left(e_{2}, s_{2}\right)$ if $\left(s_{1}, s_{2}\right) \in R_{e_{1}, e_{2}}^{D}$ and $\left(d x, s_{1}\right) \stackrel{f}{\rightarrow}\left(d x, s_{2}\right)$ if $\left(s_{1}, f\right) \in R^{C}$, where $f$ is defined on the interval $[0, t]$ and $f(t)=s_{2}$. We extend $\mathcal{R}$ to include environment steps. An environment step begins at an exit point of the mode and ends at an entry point. It represents changes to the global variables of the mode by other components while the mode is inactive. Private variables of the mode are unaffected by environment steps. Thus, there is an

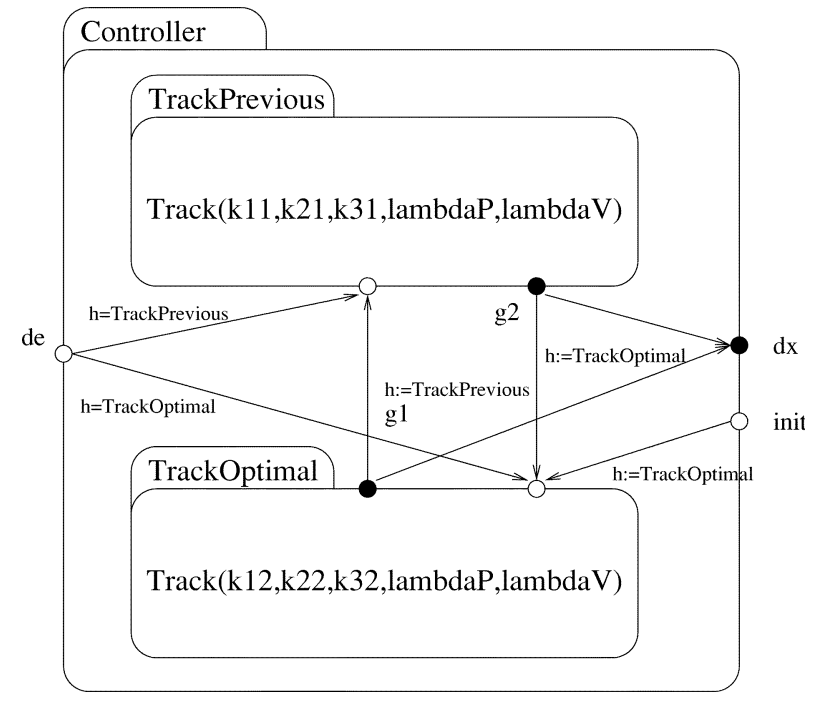

Fig. 4 The closure of a mode.

environment step $(x, s) \stackrel{\varepsilon}{\rightarrow}(e, t)$ whenever $x \in X, e \in E$ and $s\left[V_{p}\right]=t\left[V_{p}\right]$. We let $\lambda$ range over $\mathcal{F}_{V} \cup\{o, \varepsilon\}$. An execution of a mode is now a path through the graph of $\mathcal{R}$

$$
\left(e_{0}, s_{0}\right) \stackrel{\lambda_{1}}{\rightarrow}\left(e_{1}, s_{1}\right) \stackrel{\lambda_{2}}{\rightarrow} \ldots \stackrel{\lambda_{n}}{\rightarrow}\left(e_{n}, s_{n}\right) .
$$

Trace Semantics: To be able to define a refinement relation between modes, we consider trace semantics for modes. A trace of the mode is a projection of its executions onto the global variables of the mode. The trace semantics for $M$ is given by its control points $E$ and $X$, its global variables $V_{g}$, and its set of its traces $L_{M}$.

In defining compositional and hierarchical semantics, one has to decide what details of the behavior of lower-level components are observable at higher levels. In our approach, the effect of a discrete step that updates only local variables of a mode is not observable by its environment, but stoppage of time introduced by such a step is observable. For example, consider two systems, one of which is always idle, while the other updates a local variable every second. These two systems are different, since the second one does not have flows more than one second long. Defining modular semantics in a way that such distinction is not made seems much more difficult.

\section{B. Trace Semantics for Agents}

An execution of an agent $A=\langle T M, V, I\rangle$ follows a trajectory, which starts in one of the initial states and is a sequence of flows interleaved with discrete updates to the variables of the agent. An execution of $A$ is constructed from the relations $R^{C}$ and $R^{D}$ of its top-level mode. For a fixed initial state $s_{0}$, each mode $M \in T M$ starts out in the state (init ${ }_{M}, s_{M}$ ), where $\operatorname{init}_{M}$ is the nondefault entry point of $M$ and $s_{0}[M . V]=s_{M}$. Note that as long as there is a mode $M$ whose control state is at init $M$, no continuous steps are possible. However, any discrete step of such a mode will come from $R_{\text {init }_{M}, d x}^{D}$ and bring the control state of $M$ to $d x$. Therefore, any execution of the agent $A$ with $|T M|=k$ 
will start with exactly $k$ discrete initialization steps. At that point, every top-level mode of $A$ will be at its default exit point, allowing an alternation of continuous steps from $R^{C}$ and discrete steps from $R_{d e, d x}^{D}$. The choice of a continuous step involving all modes or a discrete step in one of the modes is left to the environment. Before each discrete step, there is an environment step, which takes the control point of the chosen mode from $d x$ to de and leaves all the private variables of all top-level modes intact. After that, a discrete step of the chosen mode happens, bringing control back to $d x$. Thus, an execution of $A$ with $|T M|=k$ is a sequence $s_{0} \stackrel{o}{\rightarrow} s_{1} \stackrel{\circ}{\rightarrow} \ldots s_{k} \stackrel{\lambda_{1}}{\rightarrow} s_{k+1} \stackrel{\lambda_{2}}{\rightarrow} \ldots$ such that:

1) the first $k$ steps are discrete and initialize the top-level modes of $A$.

2) for every $i \geq k$, one of the following holds:

a) the $i$ th step is a continuous step, in which every mode takes part;

b) the $i$ th step is a discrete environment step;

c) the $i$ th step is a discrete step by one of the modes and the private variables of all other modes are unchanged.

Note that environment steps in agents and in modes are different. In an agent, an environment step may contain only discrete steps, since all agents participate in every continuous step. The environment of a mode can engage in a number of continuous steps while the mode is inactive.

A trace of an agent $A$ is an execution of $A$, projected onto the set of its global variables. The denotational semantics of an agent consists of its set of global variables $V_{g}$ and its set of traces $L_{A}$.

Trace semantics for modes and agents can be related to each other in an obvious way. Given an atomic agent $A$ whose behavior is given by a mode $M$, we can obtain a trace of $A$ by taking a trace of $M$ and erasing the information about the control points from it.

\section{Compositionality Results}

As shown in [17], our semantics is compositional for both modes and agents as follows. First, the set of traces of a mode can be computed from the definition of the mode itself and the semantics of its submodes. Second, the set of traces of a composite agent can be computed from the semantics of its subagents.

Mode Refinement: The trace semantics leads to a natural notion of refinement between modes. A mode $M$ and a mode $N$ are said to be compatible if $M . V_{g}=N . V_{g}, M . E=N . E$, and $M . X=N . X$, i.e., they have the same global variables and control points. For two compatible modes $M$ and $N$, we say that $M$ refines $N$, denoted $M \preceq N$, if $L_{M} \subseteq L_{N}$, i.e., every trace of $M$ is a trace of $N$.

The refinement operator is compositional with respect to the encapsulation. If, for each submode $N_{i}$ of $M$ there is a mode $N_{i}^{\prime}$ such that $N_{i} \preceq N_{i}^{\prime}$, then we have that $M \preceq M^{\prime}$, where $M^{\prime}$ is obtained from $M$ by replacing every $N_{i}$ with $N_{i}^{\prime}$. The refinement rule is explained visually in the left side of Fig. 5.
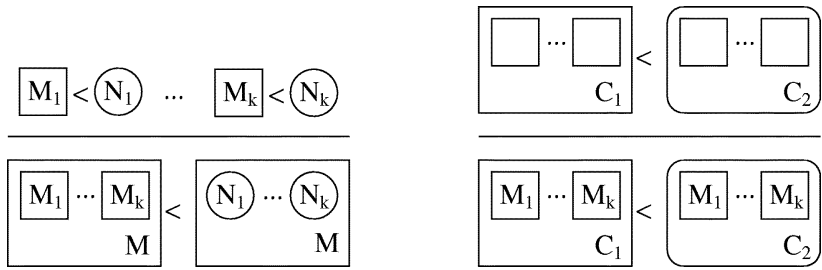

Fig. 5 Compositionality rules for modes.

A second refinement rule is defined for contexts of modes. Informally, if we consider a submode $N$ within a mode $M$, the remaining submodes of $M$ and the transitions of $M$ can be viewed as an environment or mode context for $N$.

As with modes, refinement of contexts is also defined by language inclusion and is also compositional. If a context $C_{1}$ refines another context $C_{2}$, then inserting modes $M_{1}, \ldots, M_{k}$ into the two contexts preserves the refinement property. A visual representation of this rule is shown in the right side of Fig. 5. Precise statements of the results can be found in [17].

Compositionality of Agents: An agent is, in essence, a set of top-level modes that interleave their discrete transitions and synchronize their flows. The compositionality results for modes lift in a natural way to agents too. The operations on agents are compositional with respect to refinement. An agent $A$ and an agent $B$ are said to be compatible if $A . V_{g}=B . V_{g}$. Agent $A$ refines a compatible agent $B$, denoted $A \preceq B$, if $L_{A} \subseteq L_{B}$. Given compatible agents such that $A \preceq B, A_{1} \preceq B_{1}$ and $A_{2} \preceq B_{2}$, let $V_{1}=\left\{x_{1}, \ldots, x_{n}\right\}, V_{2}=$ $\left\{y_{1}, \ldots, y_{n}\right\}$ be indexed sets of variables with $V_{1} \subseteq A . V$ and let $V_{h} \subseteq A$. $V$. Then $A \backslash\left\{V_{h}\right\} \preceq B \backslash\left\{V_{h}\right\}, A\left[V_{1}:=V_{2}\right] \preceq$ $B\left[V_{1}:=V_{2}\right]$ and $A_{1}\left\|A_{2} \preceq B_{1}\right\| B_{2}$.

\section{ANALYSIS}

Since CHARON models have a precise semantics, they can be subjected to a variety of analyzes. In this section, we give a brief overview of our ongoing research efforts in formal analysis methods for hybrid systems. These include new techniques in accurate event detection for simulation, efficient simulation, reachability analysis to detect violations of safety requirements and abstraction methods for enhancing the applicability of analysis techniques.

\section{A. Simulation Techniques}

Numerical simulation is an important tool for designing and analyzing many types of control systems, including hybrid systems. In addition to pure simulation, numerical approximation techniques are increasingly being used in reachability computations, verification, and other forms of automated analysis [33], [36], [40].

All numerical simulators operate based on some assumptions about the nature of the systems being simulated. The degree to which the system adheres to these assumptions determines how accurate the results are and what computational effort is required to generate them. Traditional numerical integration techniques typically make assumptions that tend to be violated by hybrid system models. 
In addition, the hierarchical structure of the models yields the following two observations. Often, high-level modes have very slow changing dynamics, while low-level detailed models may possess fast changing dynamics. Multiple agents in a model may be decoupled in the continuous sense, yet interact through discrete messaging. Both observations may be used to increase efficiency of simulators.

Therefore, novel simulation techniques, specific to hierarchical hybrid systems are warranted. The need for specialized simulation tools has been recognized to some degree in the literature [41], [42]. Several hybrid system simulators have been introduced (see, for example, Modelica [43], ABACUSS [44], 20-sim [45], SHIFT [22], and $\chi$ [46], as well as others reviewed in [42]). Most of the previous research has focused on properly detecting and locating discrete transitions, while largely ignoring the remaining issues. In this section, we describe three techniques that exploit the hierarchical structure of hybrid system models to provide increased accuracy and efficiency during simulation.

1) Accurate Event Detection: The problem of accurately detecting and localizing the occurrence of transitions when simulating hybrid systems has received an increased amount of attention in recent years. Formally, the event detection problem is posed as follows. Given a system

$$
\dot{s}=\left\{\begin{array}{l}
f^{M_{1}}(s), \text { if } \mathrm{g}(\mathrm{s})<0 \\
f^{M_{2}}(s), \text { if } \mathrm{g}(\mathrm{s}) \geq 0
\end{array}\right.
$$

where the mode $M \in\left\{M_{1}, M_{2}\right\}$ and $s \in Q_{M . V_{*}}$ is the continuous (or data) state, one would like to simulate the flow of $s$ according to $f^{M_{1}}$ until the first time, $t^{\prime}$, that the event $g\left(s\left(t^{\prime}\right)\right)=0$ occurs. We assume that initially $M=M_{1}$ meaning that $f^{M_{1}}$ is the active flow. Additionally we assume that the guard $g(s)<0$ is true initially.

It is generally agreed that any algorithm that addresses this problem should possess the following attributes:

1) The algorithm should be guaranteed to detect an event if one occurs and guaranteed to not return false positives.

2) If more than one event occurs in a given time interval, the algorithm ought to be capable of determining and reporting the first event.

3) Once it is determined that an event has occured, the algorithm should be able to localize precisely the time $t^{\prime}$ at which it occured.

4) Provided all of the above criteria are fulfilled, the algorithm should be as efficient as possible.

Early event detection methods, such as [47]-[50], lack rigor and are not guaranteed to correctly detect an event in many situations. More recent approaches (see [51] and [52], for example) satisfy the first three objectives in most situations while being reasonably efficient. However, a situation in which nearly all current simulators fall short is when switches occur near model singularities. Since the step-size selection scheme for the integration is typically independent of the event detection algorithm, it is entirely possible that the integrator will take a step into the region where $f^{M_{1}}(x)$ is undefined. If the particular integration method has an in-

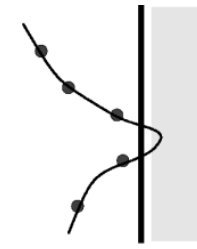

Case 1

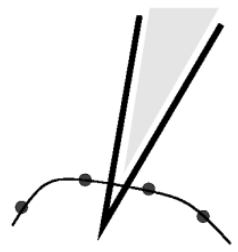

Case 2

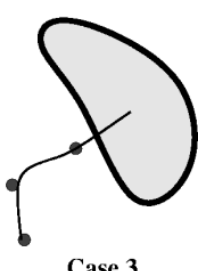

Case 3
Fig. 6 Cases 1 and 2 illustrate situations in which naive simulators can fail to detect transitions by selecting integration points which completely "miss" the guard set; Case 3 depicts a situation in which even sophisticated methods fail, when the event occurs near a region where the differential equation has a singularity at which the right side cannot be evaluated.

termediate step that requires evaluating the derivative at this state inside the singular region, a floating point exception is generated and the simulation fails abruptly. Some of these problematic situations are illustrated in Fig. 6.

We have developed a method [53] guaranteed to detect enabling of all transitions, including those occuring near singular regions. We attempt to overcome this problem by treating the event detection problem as a control system design problem. We consider the continuous dynamics of the system and the numerical integration method (we use Linear Multistep Methods_-see [54] for further details)

$$
s_{k+1}=s_{k}+h\left\{\sum_{j=1}^{m} \beta_{j} f_{k-j+1}\right\}
$$

as our collective dynamic system, where $t_{k}$ is the time of the $k$ th simulation step, $s_{k}$ is the value of the state at $t_{k}, h=$ $t_{k+1}-t_{k}$ is the simulation step size, and $\sum_{j=1}^{m} \beta_{j} f_{k-j+1}$ is some weighted combination of past values of the derivative which approximates the flow on $\left[t_{k}, t_{k+1}\right]$.

Returning to our control system analogy, the integration step size $h$ is treated as an input and the value of the transition guard, $g_{k}=g\left(s_{k}\right)$, or switching function is the output. The task at hand is to integrate the ordinary differential equation (ODE) until the boundary of the guard set is reached, taking care to never evaluate the right side of the ODE inside the guard set. In terms of our control system analogy, the problem can be rephrased as: design a feedback law that zeros the output with no overshoot. The resulting solution is essentially an Input/Output Linearization in discrete time. For a linear guard the output dynamics would be

$$
g_{k+1}=g_{k}+h \frac{\partial g}{\partial s}\left\{\sum_{j=1}^{m} \beta_{j} f_{k-j+1}\right\}
$$

selecting the step size $h$ as

$$
h=\frac{(\gamma-1) g_{k}}{\frac{\partial g}{\partial s}\left\{\sum_{j=1}^{m} \beta_{j} f_{k-j+1}\right\}}
$$

results in (5) appearing as $g_{k+1}=\gamma g_{k}$. By selecting the constant $0<\gamma<1$ we are ensured $g_{k} \rightarrow 0$ while maintaining $g_{k} \leq 0$. Thus, the simulation settles to the transition surface without overshooting it and crossing into the singular 


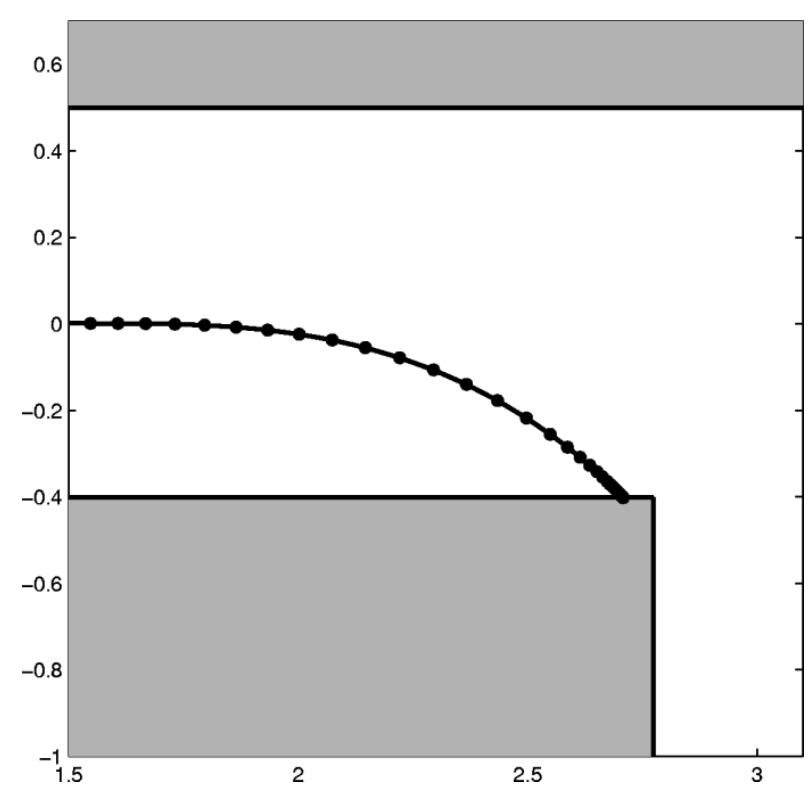

Fig. 7 The simulation takes successively smaller steps to properly locate the point at which the vehicle clips the corner.

region. This technique is illustrated in Fig. 7 where a vehicle is trying to go around a corner and the simulation must detect if it clears the corner. One can see how the simulation converges onto the exact point at which the collision occured.

2) Multirate Simulation: Many systems, especially hierarchical ones, naturally evolve on different time scales. For example, the center of mass of an automobile may be accelerating relatively slowly compared with the rate at which the crankshaft angle changes; yet the evolution of the two are intimately coupled. Despite this disparity, traditional numerical integration methods force all coupled differential equations to be integrated using the same step size. The idea behind multirate integration methods [55], [56] is to use larger step sizes for the slow changing sets of differential equations and smaller step sizes for the differential equations evolving on the fast time scale. Such a strategy increases efficiency without compromising accuracy. Areas of application include simulating integrated circuits and molecular and stellar dynamics [57]-[59]. Despite the seemingly natural connection, they have never previously been used in hierarchical hybrid systems simulation. In [60], we introduce a multirate algorithm for simulating hierarchical hybrid systems.

3) Multiagent Simulation: Multiagent hybrid systems are sets of interacting hybrid systems. In the case of the automated highway example, each vehicle may be modeled as an individual agent; however, one may like to consider the dynamics of an entire group of vehicles collectively to see how they interact. The continuous dynamics of each vehicle is physically decoupled from that of the other agents, and typically they operate independently. However, certain important discrete events may depend on the state of two or more agents. Examples of this would be when two cars come dangerously close, one car informs a group of vehicles that it is merging into the platoon, etc. Most multiagent systems of this form, when modeled in CHARON, have the following mathematical structure:

$$
\begin{aligned}
\dot{x} & =f_{x}(x) \\
\dot{y} & =f_{y}(y) \\
g(x, y) & \leq 0
\end{aligned}
$$

where $x$ and $y$ are the continuous states of agent 1 and agent 2 , their dynamics are given by the flows $f_{x}: \mathbb{R}^{n} \rightarrow \mathbb{R}^{n}$ and $f_{y}: \mathbb{R}^{m} \rightarrow \mathbb{R}^{m}$, and the predicate $g(x, y)<0$ guards a transition for one or both agents. Note that each agent's ODEs are decoupled; however, coupling is introduced through the guards.

From the point of view of simulating the continuous dynamics, it is not necessary to synchronize the integration rates of the two cars, since they are decoupled. Each set of ODEs should maximize the tradeoff between accuracy and efficiency by selecting the largest possible integration step size that is able to recreate that agents' dynamics within some acceptable user-specified error tolerance. Unfortunately, properly detecting the occurrence of events, $g(x, y)=0$, requires that the value of the state be reported in a synchronized fashion. Traditionally, simulators compute the best step size for each agent and then take the minimum as a global step size. This can result in significant inefficiencies.

Our goal is to simulate each agent with a different step size while still ensuring proper event handling. The idea is to allow the simulation for each agent to proceed independently when no events are about to occur. Only when events seem likely do we adaptively select the step sizes to bring all of the agents into synchronization to properly detect the event.

In the case of $N$ agents, our approach to this problem, reported in [61], is to define $N$ local clocks, $t_{1}, \ldots, t_{N}$ and $N$ step sizes $h_{1}, \ldots, h_{N}$, one for each agent. The step sizes are selected based on the system dynamics so as to simultaneously synchronize the local clocks and detect the event using the control theoretic technique of I/O linearization.

Fig. 8 and 9 illustrate how the simulation for two agents might proceed. Fig. 8 shows the trajectories of the two cars. The simulation tries to detect when the cars collide. Fig. 9 displays how the step sizes are selected independently throughout most of the simulation. When the system approaches an event, the local clocks automatically synchronize.

4) Distributed Simulation: The main idea behind distributed simulation is to get speedup by using multiple computing resources, since simulations of complex systems are normally very slow. Distributed simulation techniques are categorized as conservative or optimistic based on how local clocks are synchronized. If the local clock of the agent always advances and does not go backward, it is conservative simulation; otherwise, it is optimistic simulation. Conservative simulation techniques ensure that the local clock $l_{c}$ of the agent either advances or stops, but does not roll back. In optimistic simulation, the focus is to exploit possible parallelism as much as possible by allowing each agent to run at a different speed without the guarantee that 


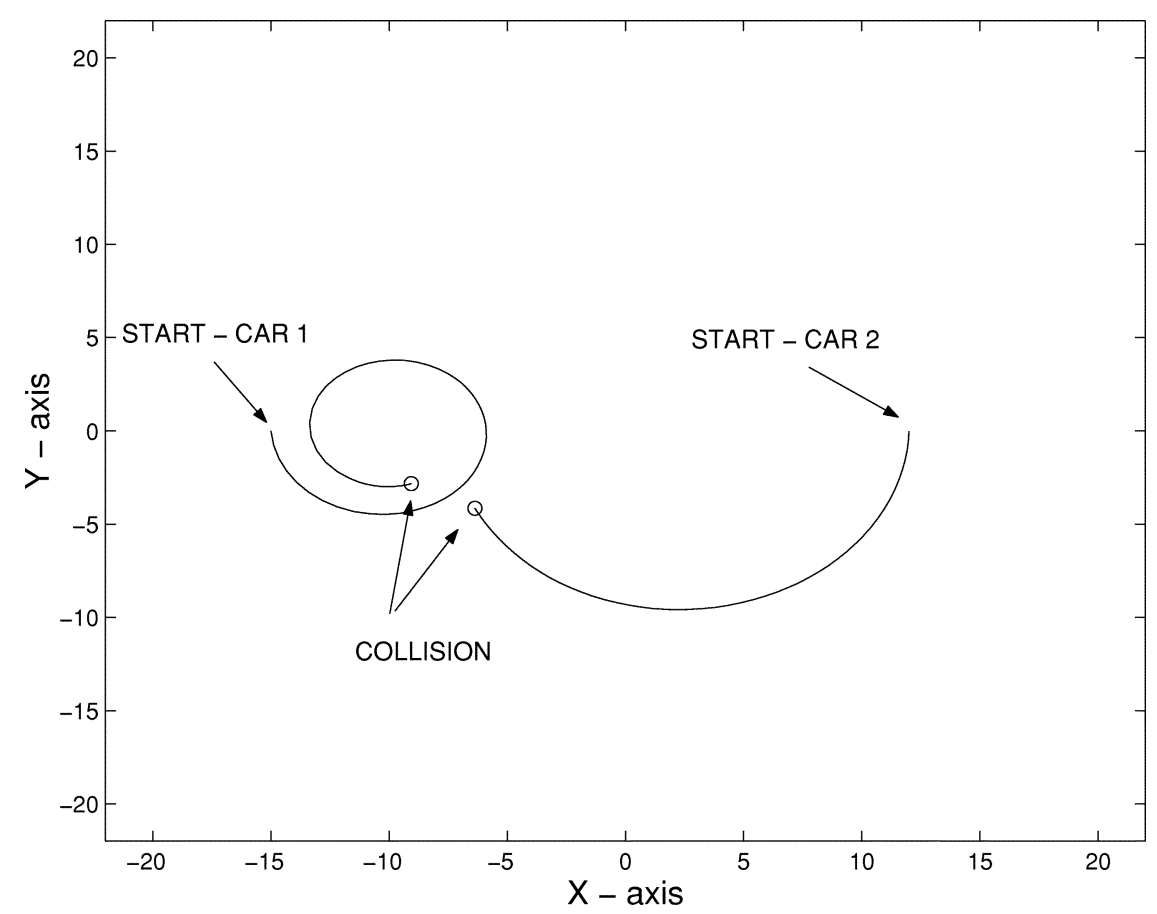

Fig. 8 The trajectories of the two cars in the plane.

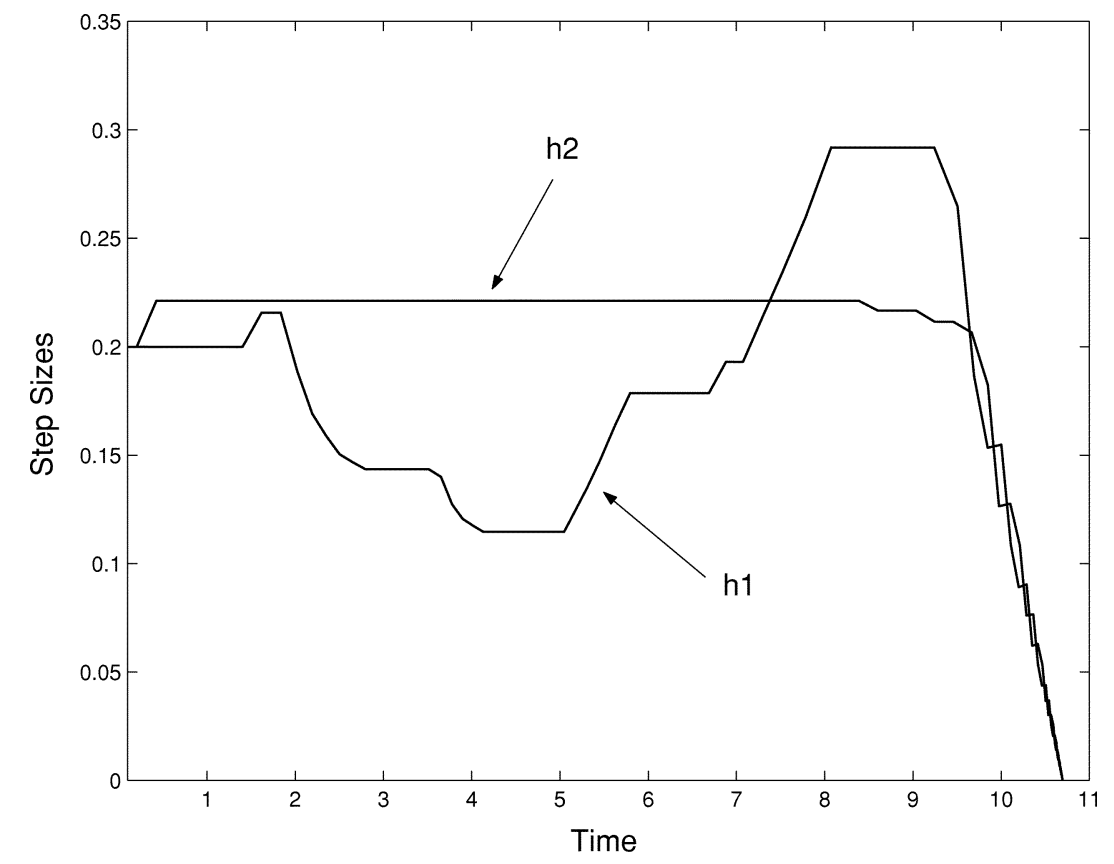

Fig. 9 Step sizes used for the two cars depicted in Fig. 8. The step sizes $h_{1}$ and $h_{2}$ are selected independently away from the constraint, but are brought into synchronization when an event is impending.

no event occurs between $t_{1}$ and $t_{2}$ when its local clock $l_{c}$ is advanced from $t_{1}$ to $t_{2}$. If an event $e$ that occurred at time $t_{e}$ gets recognized by the agent at $t_{r}$, where $t_{r}>t_{e}$, the simulator provides a rollback operation by restoring the local clock $l_{c}$ to an earlier time such that the event $e$ can be handled if and when it occurs. Note that the event $e$ may not occur at all if rollbacks are propagated to other agents so that the event $e$ becomes no longer possible.

Our approach to simulate hybrid systems in a distributed fashion is to use more computing resources by exploiting inherent modularity of systems described in CHARON. By modularity, we mean two things. One is behavioral modularity captured by mode and the other is architectural modularity by agent. One way to exploit mode-level modularity within a single agent is to use multiple rates for the simulation of the same agent as described in Section V-A2. Another way is to distribute atomic agents to exploit agent-level modularity. When the agents are distributed, they need to synchronize to update their states as the agents share information. Here, the challenge is how to reduce synchronization 


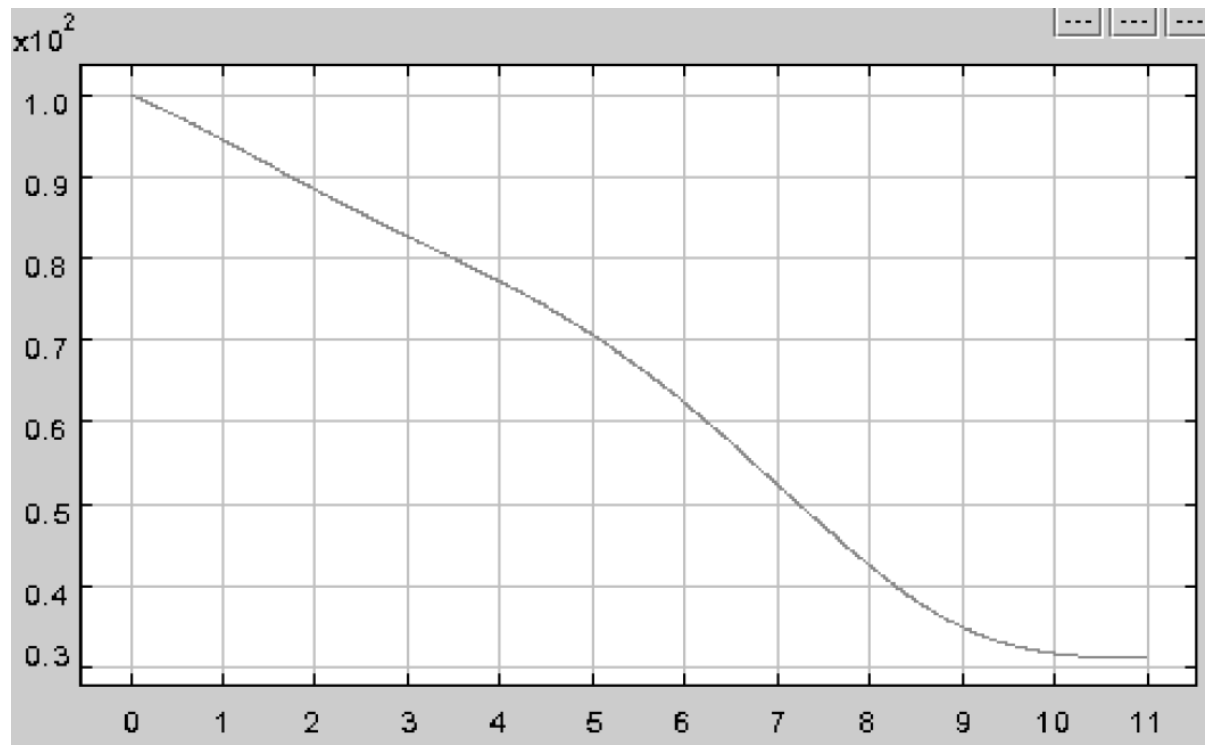

Fig. 10 The distance $d_{i}$ between the two platoons.

overheads among distributed agents. We briefly describe our conservative algorithm and optimistic algorithms.

In a conservative approach, we decompose functions into subfunctional blocks, and the simulator allows the agent to execute the next block only when all the agents complete the current block. Although our conservative approach allows to simulate hybrid systems, the disadvantage is that overhead resulting from communications degrades the possible performance gain from distributing computations. Thus, we can get speedup only in simulating very computation-oriented hybrid systems. Our optimistic simulation algorithms are to address the overhead problems. The main features of the algorithms are as follows. First, to reduce communication overhead, we let agents synchronize just before the new value of a shared variable is necessary instead of communicating every update round. Second, to reduce computation overhead due to numerical integration, we simulate the agent with its approximated polynomial dynamics and resolve the possible misses of events with a rollback operation. This allows each agent to execute its computation without integrating the shared variables controlled by other agents. Our approach is optimistic in the sense that each agent goes forward even when there is no guarantee that their clocks do not have to go backward.

5) Case Study: We now consider simulation of the platoon controller under normal conditions. Fig. 10-12 are snapshots of the CHARON plotter and show the simulation results for the following scenario. Initially, the distance between the two platoons is large, and the platoon $i$ is moving faster than the platoon in front $(i-1)$ and is therefore closing the gap. We let the velocity of the platoon in front be a sinusoidal function of time starting at an initial value 20 . One can see from the figures that the controller of platoon $i$, initially in the mode "track optimal velocity," first decreases the gap between the two platoons by accelerating. When its distance to the preceding platoon becomes small, the controller slowly decelerates and switches to mode "track velocity of previous car" approximately at time 8.2. The controller then tries to follow the platoon in front at some constant distance. Additional simulation trace plots of this example can be found in [6].

\section{B. State-Space Exploration Techniques}

1) Exact Reachability Using Requiem: Formal verification of safety requirements of hybrid systems requires the computation of reachable states of continuous systems. Requiem is a Mathematica package that, given a nilpotent linear differential equation and a set of initial conditions, symbolically computes the exact set of reachable states. Given various classes of linear differential equations and semialgebraic sets of initial conditions, the computation of reachable sets can be posed as a quantifier elimination problem in the decidable theory of reals as an ordered field [62]. Given a nilpotent system and a set defined by polynomials inequalities, Requiem automatically generates the quantifier elimination problem and invokes the quantifier elimination package in Mathematica 4.0. If the computation terminates, it returns the quantifier free formula describing the reachable set. More details can be found in [62]. The entire package is available at www.seas.upenn.edu/hybrid/requiem.html.

Parametric analysis using Requiem: We demonstrate the use of Requiem on the platoon controller described earlier. The experimental nature of the current quantifier elimination package makes it impossible to apply it to the system described by (1). We thus simplify the controller with equivalent dynamics, which controls the acceleration of the platoon $i$ instead of its derivative. This approximation results in the three dimensional system described by

$$
\left\{\begin{array}{ll}
\dot{d}_{i} & =v_{i-1}-v_{i} \\
\dot{v}_{i-1} & =a_{i-1} \\
\dot{v}_{i} & =u
\end{array} .\right.
$$

We treat the acceleration of the preceding platoon $a_{i-1}$ as a parametric disturbance and control the acceleration $\dot{v}_{i}$ of 


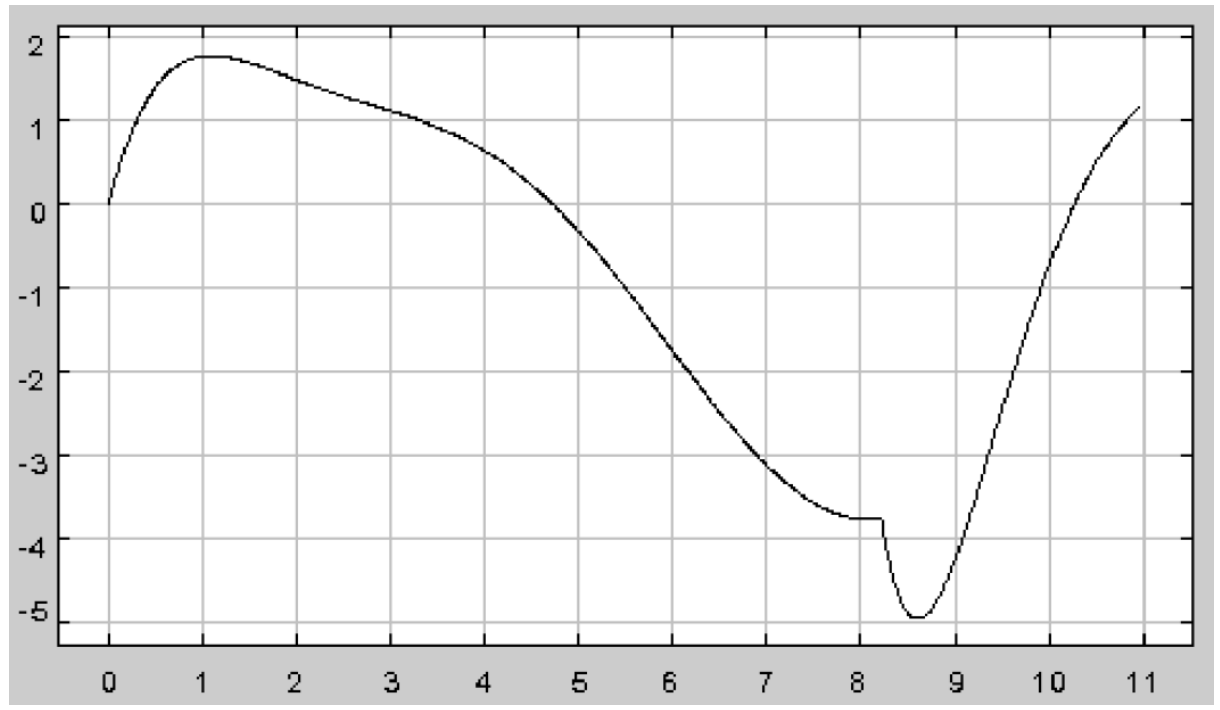

Fig. 11 The acceleration $a_{i}$ of the platoon $i$.

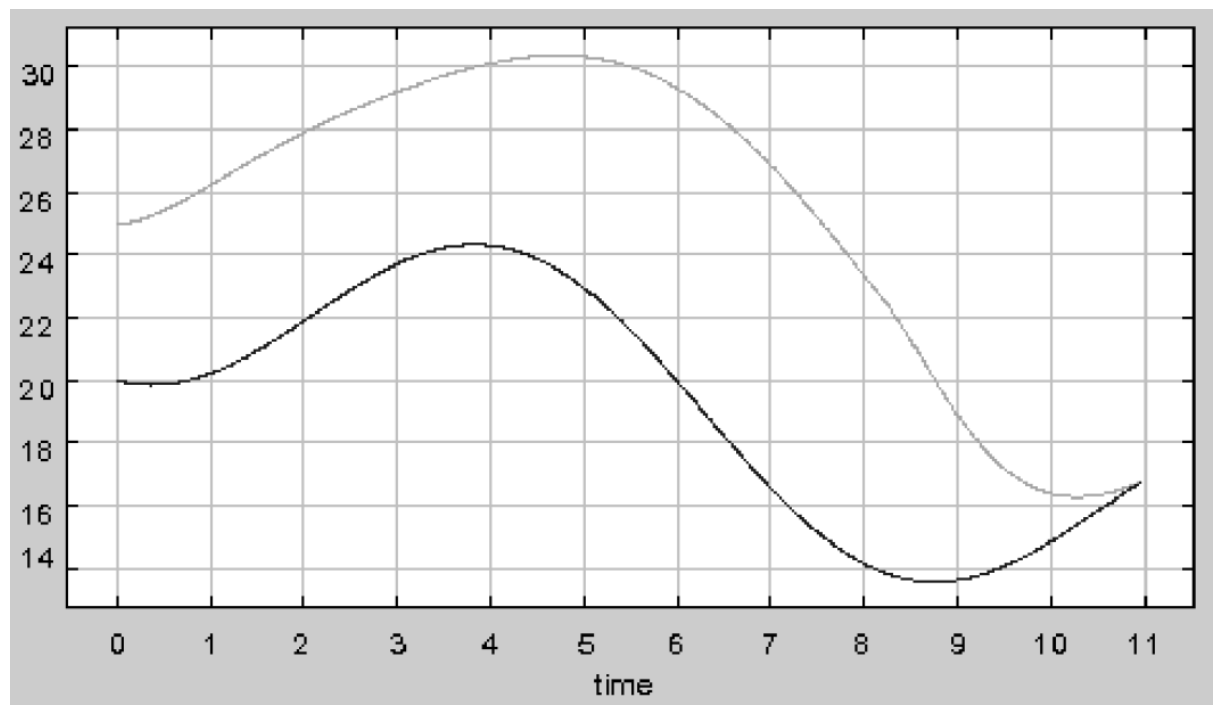

Fig. 12 The velocity of the platoon $i$ and the preceding platoon $(i-1)$ (the platoon $i$ moves faster).

the following platoon. The problem is to find the set of conditions on the parameter set $\left\{a_{i-1}, b, c\right\}$ and the state variables, which would lead to a collision $\left(d_{i} \leq 0\right)$ when we apply a control $u$ of the form $b t+c$ where $b$ and $c$ are integer constants. We use Requiem's parametric backward reachability function to obtain the quantifier free formula. By giving specific values to the parameters and initial conditions, we can see whether the formula reduces to true or false. For example, we can prove the expected result that when the vehicles are started close to each other $(d=1)$ and the control parameters $b$ and $c$ are positive, collision is unavoidable, whereas if $b$ and $c$ are negative, collision does not occur. The entire example and the output is available at www.seas.upenn.edu/hybrid/requiem/ReqIEEE.html.

2) Predicate Abstraction: In the world of program analysis, predicate abstraction has emerged to be a powerful and popular technique for extracting finite-state models from complex, potentially infinite state, discrete systems (see [63]-[66] for a sampling of this active research area).
A verifier based on this scheme requires three inputs, the (concrete) system to be analyzed, the property to be verified, and a finite set of predicates over system variables to be used for abstraction. An abstract state is a valid combination of truth values to the predicates, and thus corresponds to a set of concrete states. There is an abstract transition from an abstract state $A$ to an abstract state $B$, if there is a concrete transition from some state corresponding to $A$ to some state corresponding to $B$. The job of the verifier is to compute the abstract transitions and to search in the abstract graph looking for a violation of the property. If the abstract system satisfies the property, then so does the concrete system. If a violation is found in the abstract system, then the resulting counterexample can be analyzed to test if it is a viable execution of the concrete system. This approach, of course, does not solve the verification problem by itself. The success crucially depends on the ability to identify the "interesting" predicates, either manually or by some automated scheme and on the ability of the verifier 
to compute abstract transitions efficiently. Nevertheless, it has led to opportunities to bridge the gap between code and models and to combine automated search with user's intuition about interesting predicates. Tools such as Bandera [67], SLAM [68], and Feaver [69] have successfully applied predicate abstraction for analysis of $\mathrm{C}$ or Java programs.

Inspired by this trend, we develop algorithms for invariant verification of hybrid systems using discrete approximations based on predicate abstractions. Consider a hybrid automaton with $n$ continuous variables and a set $L$ of locations. Then the continuous state-space is $L \times \mathbb{R}^{n}$. For the sake of efficiency, we restrict our attention where all invariants, switching guards, and discrete updates of the hybrid automaton are specified by linear expressions and the continuous dynamics is linear, possibly with uncertain, bounded input. For the purpose of abstraction, the user supplies initial predicates $p_{1}, \ldots, p_{k}$, where each predicate is a polyhedral subset of $\mathbb{R}^{n}$. In the abstract program, the $n$ continuous variables are replaced by $k$ discrete Boolean variables, one Boolean variable $b_{i}$ for each predicate $p_{i}$. A combination of values to these $k$ Boolean variables represents an abstract state corresponding to a set of continuous states and the abstract state space is $L \times \mathbb{B}^{k}$. Our verifier performs an on-the-fly search of the abstract system by symbolic manipulation of polyhedra.

The core of the verifier is the computation of the transitions between abstract states that capture both discrete and continuous dynamics of the original system. Computing discrete successors is relatively straightforward and involves computing weakest preconditions and checking nonemptiness of an intersection of polyhedral sets. The implementation attempts to reduce the number of abstract states examined by exploiting the fact that each abstract state is an intersection of $k$ linear inequalities. For computing continuous successors of an abstract state $A$, we use a strategy inspired by the techniques used in CHECKMATE [33] and d/dt [34]. The basic strategy computes the polyhedral slices of states reachable from $A$ at fixed times $r, 2 r, 3 r, \ldots$ for a suitably chosen $r$, then takes the convex-hull of all these polyhedra to overapproximate the set of all states reachable from $A$. However, while tools such as CHECKMATE and d/dt are designed to compute a "good" approximation of the continuous successors of $A$, we are interested in testing if this set intersects with a new abstract state. Consequently, our implementation differs in many ways. For instance, it checks for nonempty intersection with other abstract states of each of the polyhedral slices and omits steps involving approximations using orthogonal polyhedra and termination tests (see [34]).

Postulating the verification problem for hybrid systems as a search problem in the abstract system has many benefits compared to the traditional approach of computing approximations of reachable sets of hybrid systems. First, the expensive operation of computing continuous successors is applied only to abstract states and not to intermediate polyhedra of unpredictable shapes and complexities. Second, we can prematurely terminate the computation of continuous successors whenever new abstract transitions are discovered. Finally, we can explore with different search strategies aimed at making progress in the abstract graph. For instance, our implementation always prefers computing discrete transitions over continuous ones. Our early experiments indicate that improvements in time and space requirements are significant compared to a tool such as $\mathrm{d} / \mathrm{dt}$. A more detailed description of our predicate abstraction technique for hybrid systems can be found in [70].

Verification of the platoon controller using predicate abstraction: To formally prove the safety property of this longitudinal controller, we make use of the reachability method using predicate abstraction. Here, we focus only on two regions which are critical from a safety point of view: "track optimal velocity" ( $v_{i}^{e} \leq-10$ and $\left.e_{i} \geq-1 m-\epsilon\right)$ and "track velocity of previous car" $\left(v_{i}^{e} \leq-10\right.$ and $\left.e_{i} \leq-1 m\right)$. We include a thickening parameter $\epsilon>0 \mathrm{~m}$ into the model to add nondeterminism to it. The two regions under consideration overlap allowing the controller to either use the "track optimal velocity" controller or the "track velocity of previous car" controller in this $\epsilon$-thick region. Besides adding some nondeterminism to the model, the thickening parameter also provides improved numerical stability to the simulation and reachability computation, as it is numerically hard to determine the exact time at which a switch occurs.

The respective control laws $u_{1}$ and $u_{2}$ are as follows:

$$
\begin{aligned}
& u_{1}=\frac{1}{8} d_{i}+\frac{3}{4} v_{i-1}-\left(\frac{3}{4}+\frac{1}{8} \lambda_{v}\right) v_{i}-\frac{3}{2} a_{i}-\frac{1}{8} \lambda_{p}(11) \\
& u_{2}=d_{i}+3 v_{i-1}-\left(3+\lambda_{v}\right) v_{i}-3 a_{i}-\lambda_{p} .
\end{aligned}
$$

Note that these regions correspond to situations where the platoon in front moves considerably slower; moreover, the second region is particularly safety critical because the interplatoon distance is smaller than desired.

To construct the discrete abstract system, in addition to the predicates of the invariants and guards, we include some predicates over the distance variable to be able to separate the bad region from the reachable set: $d_{i} \leq 0, d_{i} \geq 2, d_{i} \geq$ $10, d_{i} \geq 20$. The total number of initial predicates is 11 . For the initial set specified as $20 \leq d_{i} \leq 100 \wedge 15 \leq v_{i-1} \leq$ $18 \wedge 20 \leq v_{i} \leq 25$, the tool found 14 reachable abstract states and reported that the system is safe. Note this property has been proven in [71] using optimal control techniques for individual continuous modes without mode switches. Here, we prove the property for all possible behaviors of the controller.

\section{THE CHARON TOOLKIT}

In this section, we describe the CHARON toolkit. Written in Java, the toolkit features an easy-to-use graphical user interface (GUI), with support for syntax-directed text editing, a visual input language, a powerful type-checker, simulation, and a plotter to display simulation traces. The CHARON GUI uses some components from the model checker JMOCHA [72], and the plotter uses a package from the modeling tool PTOLEMY [23].

The editor windows highlight the CHARON language keywords and comments. Parsing on the fly can be enabled or disabled. In case of an error while typing, the first erroneous token 


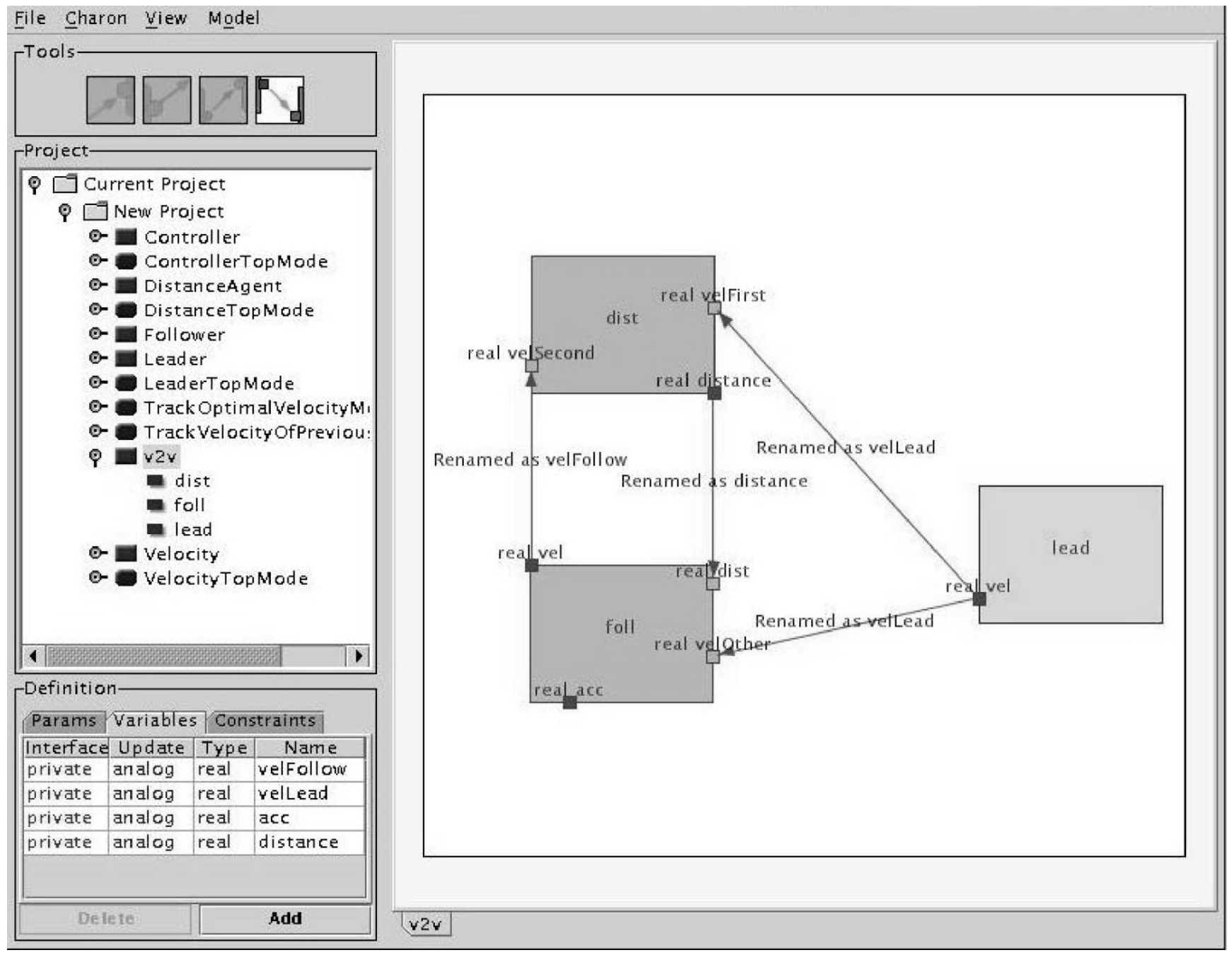

Fig. 13 The visual input tool of CHARON. The arrows depict variable renamings.

will be highlighted in red. Further, a pop-up window can be enabled that tells the user what the editor expects next. Clicking one of the pop-up options, the associated text is automatically inserted at the current cursor position. This allows the user not only to correct almost all syntactic errors at typing but also to learn the CHARON language.

The CHARON toolkit also includes a visual input language capability. It allows the user to draw agent and mode definitions at a given level of hierarchy. The visual input tool is depicted in Fig. 13, showing one level of the platoon controller from Fig. 1. By clicking on the subagents, the user can explore the lower levels of hierarchy. The interpreter of the visual input translates the specification into text-based CHARON source code using an intermediate XML-based representation.

Once a set of edited and saved CHARON language files exists, the user can simulate the hybrid system. In this case, the CHARON toolkit calls the parser and the type-checker. If there are no syntactic errors, it generates a project context that is displayed in a separate project window that appears on the left side of the desktop, as shown in Fig. 14, which displays the same model as Fig. 13.
The project window displays the internal representation of CHARON in a convenient tree format. Each node in the tree may be expanded or collapsed by clicking it. The internal representation tree consists of two nodes: agents and modes. They are initially collected from the associated CHARON files.

A CHARON specification describes how a hybrid system behaves over time. CHARON's simulator provides a means to visualize a possible behavior of the system. This information can be used for debugging or simply for understanding in detail the behavior of the given hybrid system description.

The simulation methodology used in the CHARON toolkit, which is depicted in Fig. 15, resembles concepts in code generation from a specification. Since CHARON allows the user to provide external Java source code, the simulator needs to be an executable Java program. CHARON has a set of Java files that represent a core simulator. Given a set of CHARON files, Java files are automatically generated that represent a Java interpretation of the CHARON specification of a hybrid system. They are used in conjunction with the predefined simulator core files and the external Java source code to produce a simulation trace. 


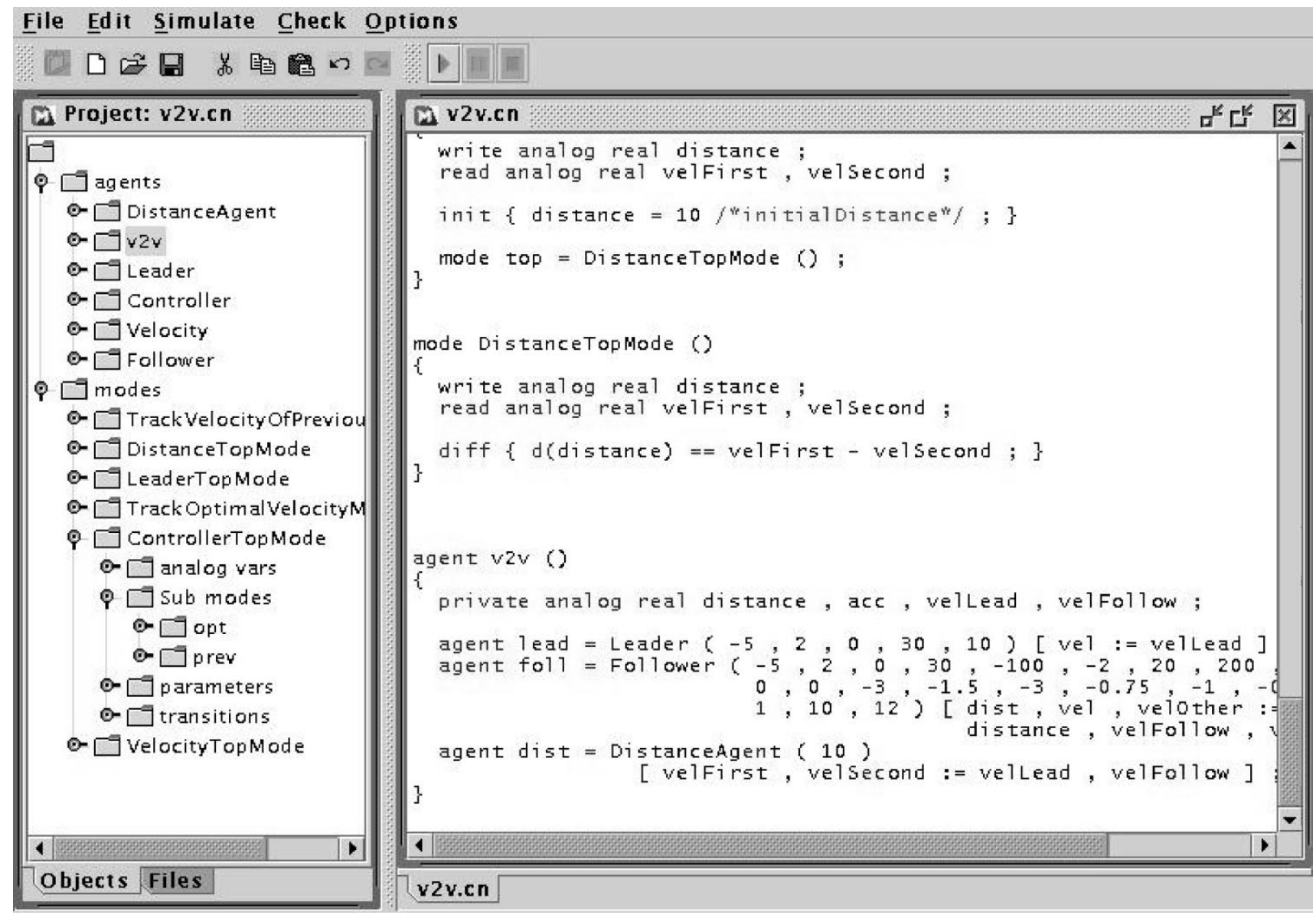

Fig. 14 The editor frame on the right side of the CHARON desktop and the corresponding project frame on the left.

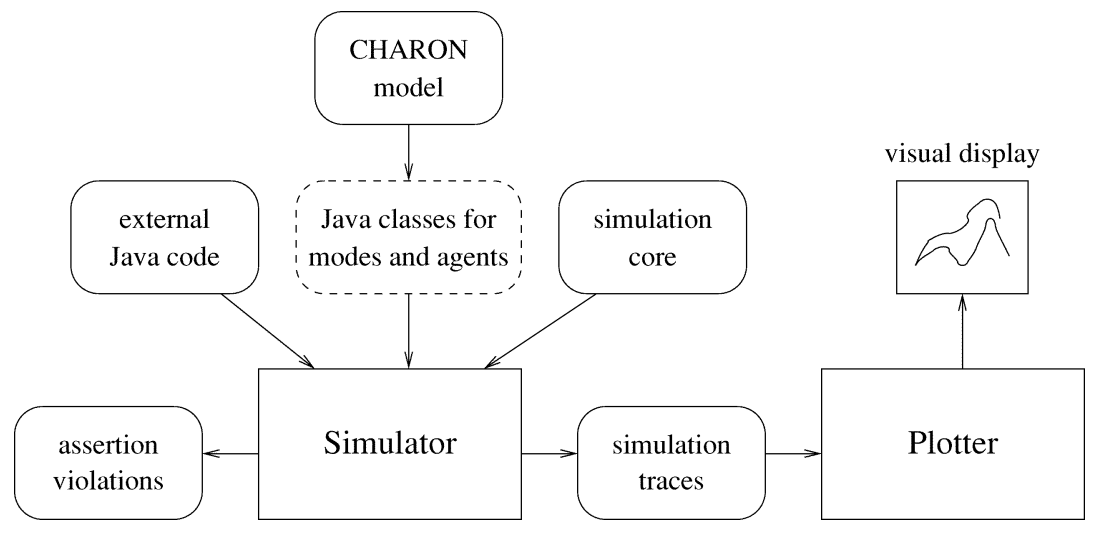

Fig. 15 The simulation methodology of CHARON.

The CHARON plotter allows the visualization of a simulation trace generated by the simulator. It draws the value of all selected variables using various colors with respect to time. It also highlights the time that selected transitions have been taken. The simulation results obtained in Fig. 10-12 have been produced using the CHARON plotter.

In addition, the simulator checks assertions that are placed in the CHARON model by the user. Assertions can be added to any mode or agent in the model. They are state predicates over the variables of the mode or agent and are supposed to be true whenever the mode is active or, for agents, always.
If an assertion is violated during a simulation, the simulator stops and the trace produced by the simulator can be used to find the source of the violation.

More information on the CHARON toolkit, along with a preliminary release, is available for free at www.cis.upenn.edu/mobies/charon/.

\section{ACKNOWLEDGMENT}

The authors would like to thank D. Huber and M. McDougall for their work on the CHARON visual interface, 
U. Sammapun for her contribution to the simulator generator, and V. Sokolskaya for the implementation of the typechecker. In addition, the authors would like to thank R. Fierro and R. Grosu for their various contributions during the initial development of CHARON.

\section{REFERENCES}

[1] E. A. Lee, "What's ahead for embedded software," IEEE Comput., pp. 18-26, Sept. 2000.

[2] R. Alur, J. Esposito, M. Kim, V. Kumar, and I. Lee, "Formal modeling and analysis of hybrid systems: A case study in multirobot coordination," in Lecture Notes in Computer Science, FM'99-Formal Methods. Heidelberg, Germany: Springer-Verlag, 1999, vol. 1708, pp. 212-232.

[3] A. Balluchi, L. Benvenuti, M. Di Benedetto, C. Pinello, and A. Sangiovanni-Vicentelli, "Automotive engine control and hybrid systems: Challenges and opportunities," Proc. IEEE, vol. 88, pp. 888-912, July 2000.

[4] C. Tomlin, G. J. Pappas, and S. Sastry, "Conflict resolution for air traffic management: A study in muti-agent hybrid systems," IEEE Trans. Automat. Contr., vol. 43, pp. 509-521, Apr. 1998.

[5] S. Engell, S. Kowalewski, C. Schulz, and O. Stursberg, "Continuousdiscrete interactions in chemical processing plants," Proc. IEEE, vol. 88, pp. 1050-1068, July 2000.

[6] F. Ivančić, "Report on Verification of the MoBIES Vehicle-Vehicle Automotive OEP Problem," Univ. of Penn., MS-CIS-02-02, 2002.

[7] R. Alur, C. Belta, F. Ivančić, V. Kumar, M. Mintz, G. Pappas, H. Rubin, and J. Schug, "Hybrid modeling and simulation of biomolecular networks," in Lecture Notes in Computer Science, Hybrid Systems: Computation and Control, M. D. Di Benedetto and A. Sangiovanni-Vincentelli, Eds. Heidelberg, Germany: Springer-Verlag, 2001, vol. 2034, pp. 19-32.

[8] E. Aaron, F. Ivančić, and D. Metaxas, "Hybrid models of navigation strategies for games and animations," in Lecture Notes in Computer Science, Hybrid Systems: Computation and Control, C. Tomlin and M. Greenstreet, Eds. Heidelberg, Germany: Springer-Verlag, 2002, vol. 2289, pp. 7-20.

[9] E. Aaron, F. Ivančić, O. Sokolsky, and D. Metaxas, "A framework for reasoning about animation systems," in Lecture Notes in Computer Science, Intelligent Virtual Agents. Heidelberg, Germany: Springer-Verlag, 2001, vol. 2190, pp. 47-60.

[10] R. Fierro, Y. Hur, I. Lee, and L. Sha, "Modeling the simplex architecture using CHARON," in Proc. 21st IEEE Real-Time Systems Symp. WIP Sessions, 2000, pp. 77-80.

[11] D. Harel, "Statecharts: A visual formalism for complex systems," Sci. Comput. Program., vol. 8, pp. 231-274, 1987.

[12] O. Maler, Z. Manna, and A. Pnueli, "From timed to hybrid systems," in Lecture Notes in Computer Science, Real-Time: Theory in Practice. Heidelberg, Germany: Springer-Verlag, 1991, vol. 600, pp. 447-484.

[13] R. Alur, C. Courcoubetis, N. Halbwachs, T. A. Henzinger, P. Ho, X. Nicollin, A. Olivero, J. Sifakis, and S. Yovine, "The algorithmic analysis of hybrid systems," Theor. Comput. Sci., vol. 138, pp. 3-34, 1995.

[14] N. Lynch, R. Segala, F. Vaandrager, and H. Weinberg, "Hybrid I/O automata," in Hybrid Systems III: Verification and Control, 1996, vol. 1066, pp. 496-510.

[15] C. A. R. Hoare, Communicating Sequential Processes. Englewood Cliffs, NJ: Prentice-Hall, 1985.

[16] R. Milner, Lecture Notes in Computer Science, A Calculus of Communicating Systems. Heidelberg, Germany: Springer-Verlag, 1980, vol. 92.

[17] R. Alur, R. Grosu, I. Lee, and O. Sokolsky, "Compositional refinement for hierarchical hybrid systems," in Lecture Notes in Computer Science, Hybrid Systems: Computation and Control. Heidelberg, Germany: Springer-Verlag, 2001, vol. 2034, pp. 33-48.

[18] G. Booch, I. Jacobson, and J. Rumbaugh, Unified Modeling Language User Guide. Boston, MA: Addison-Wesley, 1997.

[19] S. E. Mattsson and M. Anderson, "The ideas behind omola," in CACSD 92: IEEE Symp. Comput. Aided Control Syst. Design, 1992, pp. 23-29.

[20] H. Elmqvist, F. E. Cellier, and M. Otter, "Object-oriented modeling of hybrid systems," in Proc. Eur. Simulation Symp., 1993, pp. 31-41.
[21] H. Elmqvist, S. E. Mattsson, and M. Otter, "Modelica-The new object-oriented modeling langugae," in Proc. 12th Eur. Simulation Multiconf., 1998, pp. 127-131.

[22] A. Deshpande, A. Gollu, and L. Semenzato, "The Shift Programming Language and Run-Time System for Dynamic Networks of Hybrid Automata," Univ. California Berkeley, UCB-ITS-PRR-97-7, 1997.

[23] J. Davis, M. Goel, C. Hylands, B. Kienhuis, E. A. Lee, J. Liu, X. Liu, L. Muliadi, S. Neuendorffer, J. Reekie, N. Smyth, J. Tsay, and Y. Xiong, "Overview of the Ptolemy Project," Univ. California Berkeley, UCB/ERL M99/37, 1999.

[24] E. M. Clarke and R. P. Kurshan, "Computer-aided verification," IEEE Spectr., vol. 33, pp. 61-67, June 1996.

[25] G. J. Holzmann, "The model checker SPIN," IEEE Trans. Software Eng., vol. 23, pp. 279-295, May 1997.

[26] R. Alur and D. L. Dill, "A theory of timed automata," Theor. Comput. Sci., vol. 126, pp. 183-235, 1994.

[27] R. Alur, T. Henzinger, G. Lafferriere, and G. Pappas, "Discrete abstractions of hybrid systems," Proc. IEEE, vol. 88, pp. 971-984, July 2000.

[28] N. Halbwachs, Y. Proy, and P. Raymond, "Verification of linear hybrid systems by means of convex approximations," in Lecture Notes in Computer Science, Static Analysis. Heidelberg, Germany: Springer-Verlag, 1994, vol. 864.

[29] T. A. Henzinger, "The theory of hybrid automata," in Proc. 11th IEEE Symp. Logic in Comput. Sci., 1996, pp. 278-293.

[30] C. Daws, A. Olivero, S. Tripakis, and S. Yovine, "The tool KRONOS," in Lecture Notes in Computer Science, Hybrid Systems III: Verification and Control. Heidelberg, Germany: Springer-Verlag, 1996, vol. 1066, pp. 208-219.

[31] K. Larsen, P. Pettersson, and W. Yi, "UPPAAL in a nutshell," Springer Int. J. Softw. Tools Technol. Transfer, vol. 1, 1997.

[32] T. A. Henzinger, P. Ho, and H. Wong-Toi, "Hytech: The next generation," in Lecture Notes in Computer Science, Tools and Algorithms for the Construction and Analysis of Systems. Heidelberg, Germany: Springer-Verlag, 1995, vol. 1019, pp. 41-71.

[33] A. Chutinan and B. K. Krogh, "Verification of polyhedral-invariant hybrid automata using polygonal flow pipe approximations," in Lecture Notes in Computer Science, Hybrid Systems: Computation and Control. Heidelberg, Germany: Springer-Verlag, 1999, vol. 1569, pp. 76-90.

[34] E. Asarin, O. Bournez, T. Dang, and O. Maler, "Approximate reachability analysis of piecewise-linear dynamical systems," in Lecture Notes in Computer Science, Hybrid Systems: Computation and Control. Heidelberg, Germany: Springer-Verlag, 2000, vol. 1790, pp. 21-31.

[35] M. Greenstreet and I. Mitchell, "Reachability analysis using polygonal projections," in Lecture Notes in Computer Science, Hybrid Systems: Computation and Control. Heidelberg, Germany: Springer-Verlag, 1999, pp. 103-116.

[36] I. Mitchell and C. Tomlin, "Level set methods for computation in hybrid systems," in Lecture Notes in Computer Science, Hybrid Systems: Computation and Control. Heidelberg, Germany: SpringerVerlag, 2000, vol. 1790, pp. 310-323.

[37] A. Kurzhanski and P. Varaiya, "Ellipsoidal techniques for reachability analysis," in Lecture Notes in Computer Science, Hybrid Systems: Computation and Control. Heidelberg, Germany: SpringerVerlag, 2000, vol. 1790, pp. 202-214.

[38] P. Varaiya, "Smart cars on smart roads: Problems of control," IEEE Trans. Automat. Contr., vol. 38, Feb. 1993.

[39] D. Godbole and J. Lygeros, "Longitudinal control of a lead card of a platoon," IEEE Trans. Veh. Technol., vol. 43, no. 4, pp. 1125-1135, 1994.

[40] T. Dang and O. Maler, "Reachability analysis via face lifting," in Lecture Notes in Computer Science, Hybrid Systems: Computation and Control, T. Henzinger and S. Sastry, Eds. Heidelberg, Germany: Springer-Verlag, 1998, vol. 1386, pp. 96-109.

[41] S. Kowalewski, M. Fritz, H. Graf, J. Preubig, S. Simon, O. Stursberg, and $\mathrm{H}$. Treseler, "A case study in tool-aided analysis of discretely controled continuous systems: The two tanks problem," in Lecture Notes in Computer Science, Hybrid Systems V. Heidelberg, Germany: Springer-Verlag, 1999, vol. 1567.

[42] P. Mosterman, "An overview of hybrid simulation phenomena and their support by simulation packages," in Hybrid Systems: Computation and Control, F. Varager and J. H. van Schuppen, Eds. Heidelberg, Germany: Springer-Verlag, 1999, vol. 1569, pp. 163-177. 
[43] P. Fritzson and V. Engelson, "Modelica-A unified object-oriented language for system modeling and simulation," in ECOOP 98: The 12th Eur. Conf. Object-Oriented Program., 1998, pp. 67-90.

[44] R. Allgor, P. Barton, and W. Feehery, "A large scale differentialalgebraic and parametric sensitivity solver," ABACUSS Project Rep., vol. 97, no. 1, pp. 1-4, 1997.

[45] J. Broenink, "Modeling, simulation and analysis with 20-sim," Journal A, vol. 38, no. 3, pp. 22-25, 1995.

[46] D. van Beck, G. Fabian, and J. Rooda, "Integration of the discrete and the continuous behavior in the hybrid chi simulator," in Proc. 1998 Eur. Simulation Multiconf., 1998, pp. 252-257.

[47] M. B. Carver, "Efficient integration over discontinuities in ordinary differential equation simulations," Math. and Comput. Simulation, vol. XX, pp. 190-196, 1978.

[48] F. Cellier, "Combined discrete/continuous system simulation by use of digital computers: techniques and tools," Ph.D. dissertation, ETH Zurich, Zurich, Switzerland, 1979.

[49] C. W. Gear and O. Osterby, "Solving ordinary differential equations with discontinuities,” Dept. Comput. Sci., Univ. Ill., 1981.

[50] L. F. Shampine, I. Gladwell, and R. W. Brankin, "Reliable solution of special event location problems for ODE's," ACM Trans. Math. Softw., vol. 17, no. 1, pp. 11-25, Mar. 1991.

[51] V. Bahl and A. Linninger, "Modeling of continuous-discrete processes," in Hybrid Systems: Computation and Control. Heidelberg, Germany: Springer-Verlag, 2001, vol. 2034, pp. $387-402$.

[52] T. Park and P. Barton, "State event location in differential-algebraic models," ACM Trans. Model. Comput. Simulation, vol. 6, no. 2, pp. 137-165, 1996.

[53] J. Esposito, V. Kumar, and G. Pappas, "Accurate event detection for simulating hybrid systems," in Hybrid Systems: Computation and Control. Heidelberg, Germany: Springer-Verlag, 2001, vol. 2034, pp. 204-217.

[54] U. Ascher and L. Petzold, Computer Methods for Ordinary Differential Equations and Differential-Algebraic Equations. Philadelphia, PA: Soc. for Ind. and Applied Math., 1998.

[55] C. W. Gear and D. R. Wells, "Multirate linear multistep methods," BIT, vol. 24, pp. 484-502, 1984.

[56] R. C. Rice, "Split Runge-Kutta methods for simultaneous equations," J. Res. Nat. Bur. Stand., vol. 64B, pp. 151-170, 1960.

[57] C. Engstler and C. Lubich, "Multirate extrapolation methods for differential equations with different time scales," Computing, vol. 58, pp. 173-185, 1996.

[58] M. Gunther and P. Rentrop, "Multirate row methods and latency of electrical circuits," Appl. Numer. Math., vol. 13, pp. 83-102, 1993.

[59] J. Sand and S. Skelboe, "Stability of backward euler multirate methods and convergence of waveform relaxation," BIT, vol. 32, pp. 350-366, 1992.

[60] J. Esposito and V. Kumar, "Efficient dynamic simulation of robotic systems with hierarchy," in IEEE Int. Conf. Robotics Automation, May 2001, pp. 2818-2823.

[61] J. Esposito, G. Pappas, and V. Kumar, "Multi-agent hybrid system simulation," in Proc. 40th IEEE Conf. Decision and Contr., Dec. 2001, pp. 780-786.

[62] G. Lafferriere, G. Pappas, and S. Yovine, "Symbolic reachability computation for families of linear vector fields," J. Symbol. Comput., vol. 32, no. 3, pp. 231-253, Sept. 2001.

[63] E. Clarke, O. Grumberg, S. Jha, Y. Lu, and H. Veith, "Counterexample-guided abstraction refinement," in Lecture Notes in Computer Science, Computer Aided Verification. Heidelberg, Germany: Springer-Verlag, 2000, vol. 1855, pp. 154-169.

[64] P. Cousot and R. Cousot, "Abstract interpretation: A unified lattice model for static analysis of programs by construction or approximation of fixpoints," in Proc. 4th ACM Symp. Principles Program. Lang., 1977, pp. 238-252.

[65] S. Das, D. Dill, and S. Park, "Experience with predicate abstraction," in Computer Aided Verification, 1999, vol. 1633, pp. 160-171.

[66] S. Graf and H. Saidi, "Construction of abstract state graphs with PVS," in Computer Aided Verification , 1997, vol. 1254, pp. 72-83.

[67] J. C. Corbett, M. B. Dwyer, J. Hatcliff, S. Laubach, C. S. Pasareanu, Robby, and H. Zheng, "Bandera: Extracting finite-state models from Java source code," in Proc. 22nd Int. Conf. Software Eng., 2000, pp. $439-448$.
[68] T. Ball and S. Rajamani, "Bebop: A symbolic model checker for Boolean programs," in Lecture Notes in Computer Science, SPIN Model Checking and Software Verification. Heidelberg, Germany: Springer-Verlag, 2000, vol. 1885, pp. 113-130.

[69] G. J. Holzmann and M. H. Smith, "Automating software feature verification,” Bell Labs Tech. J., vol. 5, no. 2, pp. 72-87, 2000.

[70] R. Alur, T. Dang, and F. Ivančić, "Reachability analysis of hybrid systems via predicate abstraction," in Lecture Notes in Computer Science, Hybrid Systems: Computation and Control, C. Tomlin and M. Greenstreet, Eds. Heidelberg, Germany: Springer-Verlag, Mar. 2002, vol. 2289, pp. 35-48.

[71] A. Puri and P. Varaiya, "Driving Safely in Smart Cars," Cal. PATH, Univ. Cal., Berkeley, UBC-ITS-PRR-95-24, 1995.

[72] R. Alur, L. de Alfaro, R. Grosu, T. A. Henzinger, M. Kang, R. Majumdar, F. Mang, C. M. Kirsch, and B. Y. Wang, "Mocha: A model checking tool that exploits design structure," in Proc. 23rd Int. Conf. Softw. Eng., 2001, pp. 835-836.

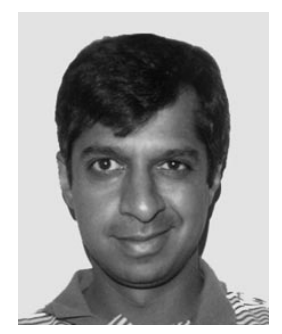

Rajeev Alur (Member, IEEE) received the B.Tech. degree in computer science from the Indian Institute of Technology, Kanpur, India, in 1987 and the Ph.D. degree in computer science from Stanford University, Stanford, CA, 1991.

He was previously with Bell Laboratories, Lucent Technologies, Murray Hill, NJ. He is currently a Professor of Computer and Information Science at the University of Pennsylvania, Philadelphia. He has published more than 70 articles in refereed journals and conference proceedings and served on numerous scientific committees. He serves on the editorial board of Formal Methods in System Design, Kluwer. His research interests include software engineering, design automation for embedded systems, and applied formal methods. He is well known for his research on timed and hybrid automata, a framework for specification and analysis of real-time systems.

Dr. Alur has won such awards as the Sloan Faculty Fellowship and the NSF CAREER award. He co-organized and cochaired the 1995 Workshop on Hybrid Systems and the 1996 Conference on Computer-Aided Verification.

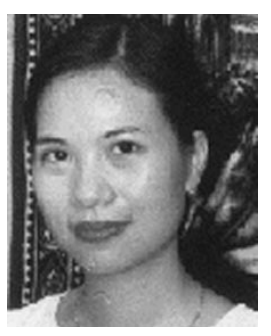

Thao Dang received the Diplôme d'Ingénieur degree and the M.Sc. degree in electrical engineering from Ecole Nationale Supérieure d'Ingénieurs Electriciens, Grenoble, France, in 1996. She received the Ph.D. degree in automatic control from the Verimag Laboratory, Grenoble, France, in 2000.

From 2001 to 2002, she was a Postdoctoral Research Associate at the Department of Computer and Information Science, University of Pennsylvania, Philadelphia. She is currently a research scientist at the Centre National de la Recherché Scientifique (CNRS) (the French National Center of Scientific Research) and a member of the Verimag Laboratory. Her research interests are modeling, verification, and control of hybrid systems, and their applications in design and analysis of embedded real-time systems.

Joel Esposito is currently a Ph.D. degree candidate at the General Robotic and Active Sensory Perception (GRASP) Lab at the University of Pennsylvania, Philadelphia.

His research interests include simulation of hybrid and embedded systems as well as developing other numerical and computational tools for control system design. He also works on motion planning and control for mobile robotics. 


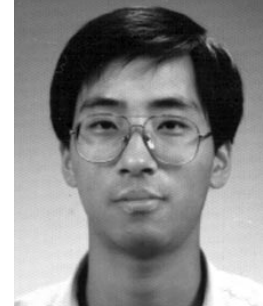

Yerang Hur received the B.S. and M.S. degrees in computer engineering from Seoul National University, Seoul, South Korea, in 1994 and 1996, respectively. He is currently a Ph.D. degree candidate in the Department of Computer and Information Science, University of Pennsylvania, Philadelphia.

His research interests are in the areas of design automation, modeling and analysis of hybrid systems, parallel and distributed simulation, programming language support for system designs, real-time operating systems, mobile computing, distributed systems, and computer architecture.

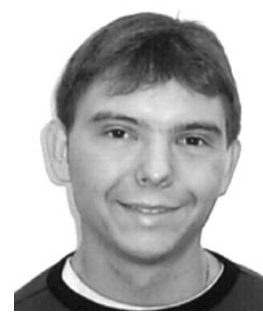

Franjo Ivančić (Student Member, IEEE) received the Diplom degree in computer science from Rheinische Friedrich-Wilhelms University, Bonn, Germany, in 1999 and the M.S.E. degree in computer science from the University of Pennsylvania, Philadelphia, in 2000.

From 1997 to 1999, he was a Research Associate at the German National Research Center for Information Technology, Sankt Augustin, Germany. He is currently a Ph.D. candidate in the Department of Computer and Information Science, University of Pennsylvania. His research interests include software design automation techniques for embedded systems and formal methods for the analysis of hybrid systems.

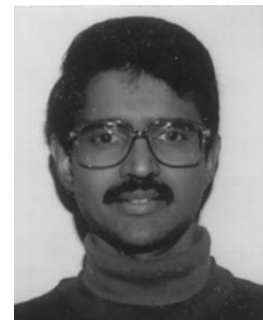

Vijay Kumar received the M.Sc. and Ph.D. degrees in mechanical engineering from Ohio State University, Columbus, in 1985 and 1987, respectively.

From 1987 to present, he has been on the faculty in the Department of Mechanical Engineering and Applied Mechanics with a secondary appointment in the Department of Computer and Information Science at the University of Pennsylvania, Philadelphia. He is currently a Professor and the Deputy Dean for research in the School of Engineering and Applied Science. He has served on the Editorial Board of the Journal of Franklin Institute and the ASME Journal of Mechanical Design. His research interests include robotics, dynamics, control, design, and biomechanics.

Dr. Kumar is a member of the American Society of Mechanical Engineers and the Robotics International Society of Manufacturing Engineers. $\mathrm{He}$ is the recipient of the 1991 National Science Foundation Presidential Young Investigator award and the 1997 Freudenstein Award for significant accomplishments in mechanisms and robotics. He has served on the editorial board of the IEEE TRANSACTIONS ON ROBOTICS AND AUTOMATION.

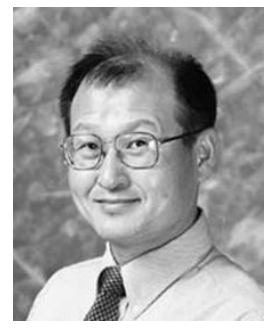

Insup Lee (Fellow, IEEE) received the B.S. degree in mathematics from the University of North Carolina, Chapel Hill, in 1977 and the Ph.D. degree in computer science from the University of Wisconsin, Madison, in 1983.

From 1983 to present, he has been with the Department of Computer and Information Science, University of Pennsylvania, Philadelphia. $\mathrm{He}$ is currently Professor in that department. He has been on the editorial boards of Formal Methods in System Design and Journal of Electrical Engineering and Information Science. His research interests include real-time systems, formal methods, and software engineering. $\mathrm{He}$ has been developing programming concepts, language constructs, and operating systems for real-time systems. In recent years, he has been developing specification, analysis, and testing techniques based on real-time process algebra (ACSR) and hybrid systems. Furthermore, he has been implementing and evaluating software engineering tools based on formal techniques. He has also developed the $\mathrm{MaC}$ framework, which can be used to assure the correctness of a running system through monitoring and checking of safety properties.

Dr. Lee has been on the editorial board of IEEE TRANSACTIONS ON COMPUTERS. From 1994 to 1997, he was Computer Science and Engineering Undergraduate Curriculum Chair at the University of Pennsylvania. He has served on numerous program committees and has also chaired or cochaired several conferences and workshops.

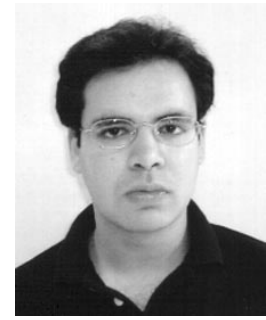

Pradyumna Mishra received the B.Tech. degree from the Department of Electrical Engineering at the Indian Institute of Technology, Kharagpur, India, in 2000.

$\mathrm{He}$ is currently a Ph.D. degree candidate in the Department of Computer and Information Science at the University of Pennsylvania, Philadelphia. His research interests are in the areas of embedded control systems, hybrid systems, distributed robotics, and air traffic management systems.

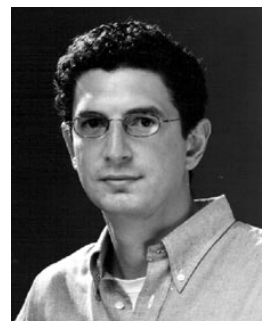

George J. Pappas (Member, IEEE) received the B.S. degree in computer and systems engineering and the M.S. degree in computer and systems engineering from the Rensselaer Polytechnic Institute, Troy, NY, in 1991 and 1992, respectively. He received the Ph.D. degree from the Department of Electrical Engineering and Computer Sciences, University of California, Berkeley, in 1998.

In 1994, he was a Graduate Fellow at the Division of Engineering Science of Harvard University, Cambridge, MA. He is currently an Assistant Professor and Graduate Group Chair in the Department of Electrical Engineering at the University of Pennsylvania, Philadelphia, where he also holds a Secondary Appointment in the Department of Computer and Information Sciences. His research interests include embedded hybrid systems, hierarchical control systems, nonlinear control systems, geometric control theory, flight and air traffic management systems, robotics, and unmanned aerial vehicles.

Dr. Pappas is the recipient of the 2002 NSF CAREER award and the 1999 Eliahu Jury Award for Excellence in Systems Research from the Department of Electrical Engineering and Computer Sciences at the University of California, Berkeley.

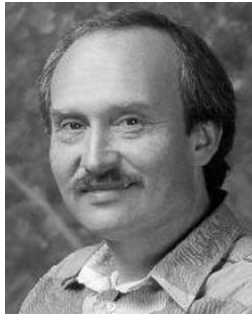

verification
Oleg Sokolsky received the M.Sc. degree in computer science from St. Petersburg Technical University, St. Petersburg, Russia, in 1988 and the $\mathrm{Ph} . \mathrm{D}$. degree in computer science from the State University of New York, Stony Brook, in 1996.

$\mathrm{He}$ is currently a Research Assistant Professor at the University of Pennsylvania, Philadelphia, where he has held research staff positions since 1998. His research interests include formal methods for the analysis of real-time and hybrid systems, model checking, and run-time 